\title{
Quantum Chemical Studies on Structural, Spectroscopic, Thermochemistry, Photo-physical and Bioactivity Properties of m-Cresol Purple Dye
}

\author{
Mohamed A.M. El-Mansy ${ }^{1,2}{ }^{(\mathbb{D})}$, Athisaya Suvitha ${ }^{3(\mathbb{D})}$, Medhat Ibrahim ${ }^{4, * \mathbb{C}}$ \\ 1 Molecular Modeling Simulation Lab., Department of Physics, Faculty of Education, Ain Shams University, Roxy, Cairo, \\ Egypt \\ 2 Condensed Matter Theory Group, Department of Physics, College of Science and Arts, Qassim University, Ar Rass, 51921, \\ Saudi Arabia \\ 3 Department of Physics, CMR Institute of Technology, Bangalore, Karnataka, India \\ 4 Molecular Spectroscopy and Modeling Unit, Spectroscopy Department, National Research Centre, 33 El-Bohouth St., \\ 12622 Dokki, Giza, Egypt \\ * Correspondence: ma.khalek@nrc.sci.eg;
}

Scopus Author ID 8641587100

Received: 15.02.2021; Revised: 5.04.2021; Accepted: 9.04.2021; Published: 26.04.2021

\begin{abstract}
The m-Cresol purple molecule is analyzed using spectroscopy and quantum computational chemistry methods using the software program Gaussian 09. B3LYP 6-311G $(\mathrm{d}, \mathrm{p})$ level has been used to create stable conformation of molecular structure, vibrational frequencies, Mulliken atomic charges, and electronic absorption spectra. The active regions of the infrared intensities, polarizabilities, and first-order polarizabilities were determined. The visible ultrasonic absorption with DOS spectrum demonstrated the highest correlation both before and after UV exposure. Furthermore, 'Frontier's molecular orbital analysis was determined, explaining the difference between HOMOs and LUMOs energies. Swiss ADME is used to measure physicochemical descriptors and the prediction of molecular dynamics, ADME (absorption, distribution, metabolism, excretion) coefficients, pharmaco-kinetics pH, $\log$ P, biological activity, and drug-like nature. Furthermore, the predictive model of BOILED-Egg, QSAR analysis, molecular lipophilicity, distribution of microorganisms, target binding percentages, and topology measurements are analyzed to help drug discovery.
\end{abstract}

Keywords: band offsets; thermochemistry, nonlinear optics; ADME; QSAR.

(C) 2021 by the authors. This article is an open-access article distributed under the terms and conditions of the Creative Commons Attribution (CC BY) license (https://creativecommons.org/licenses/by/4.0/).

\section{Introduction}

Worldwide seek urged scientists' core researches to fabricate novel efficient solar cells [1,2]. Recent solar devices are mainly based on organic materials due to affordable fabrication, outstanding potential efficiencies [3, 4], and the ease of photocurrent enhancement upon tailoring their molecular structure [5,6]. Meta-cresol purple $(\mathrm{m}-\mathrm{CP})$ has been used in seawater $\mathrm{pH}$ column assessments in various ocean zones [7,8]. Seawater $\mathrm{pH}$ characterizes the ocean's acid-based framework upon certain equilibrium circumstances (salinity, temperature, pressure) [9]. $\mathrm{pH}$ values index the oceanic $\mathrm{CO}_{2}$ system, reflecting major procedures like production and respiration, air-sea $\mathrm{CO}_{2}$ exchange, and $\mathrm{CaCO}_{3}$ dissolution. $\mathrm{m}-\mathrm{CP}$ has a chemical formula $\mathrm{C}_{21} \mathrm{H}_{18} \mathrm{O}_{5}$ Sand its molecular mass is 382.0875 a.m.u, as shown in Fig.1-a. The Molecular simulation technique recently turned into a unique tool to foresee both structural, spectral, and thermochemical features through computational-based programs. Authors used molecular simulation as significant spectroscopic confirmation means for their experimental FT-IR 
spectra [10]. Density functional principles (DFT) using Becke`s three functionals with Lee, Yang \& Parr (B3LYP) selecting 6-311G level is an excellent well-known fashion for computing FTIR spectra [11-19] as well as complex heteromoties bonding and conducting polymers optoelectronic aspects [14] whereas WB97XD6-311G level is used for nonlinear optics (NLO) calculations.

As recently reported, m-CP structural, electronic, optical, and nonlinear optical (NLO) properties have not been published yet. Accordingly, such a study will be a step towards investigating $\mathrm{m}-\mathrm{CP}$ characteristics in advance.

\section{Materials and Methods}

\subsection{Computational details.}

A theoretical approach is carried out through collaborated Gaussian (G09) [20] with Gauss View 5 (GV5) [21] software considering the B3LYP6-311G set. m-CP optimum geometry, FTIR charts, HOMO LUMO bandgap, ionized-potential IP, electron-affinity EA, chemical-potential $\chi$, hardness $\kappa$, electrophile-index $\phi$, softness $S$ are computed by the same trend. Also, m-CP NLO response is checked using the WB97XD6-311G set. The density of state charts is resolved through Gauss Sum 3. Drug bank computation carried out the QSAR study, the molecular lipophilicity of m-CP [22-24]. ADME parameters, pharmacophore modeling, pharmaco-kinetics / pharmaco-dynamics, metabolism, Boiled-Egg predictive model, biological activity radar, and target binding percentage were performed using Swiss ADME software [25-27]. The distribution of microorganisms and topology measurements are also determined by the Marvin sketch program [28-30] to analyze the active region of the compound.

\section{Results and Discussion}

\subsection{Optimum geometry.}

Computed B3LYP6-311G crystallographic parameters for m-CP are registered in Table 1. Inasmuch as crystallographic data paucity on $\mathrm{m}-\mathrm{CP}$, experimental/theoretical crystal report will not be registered in the meantime. According to optimal m-CP geometry (see Fig.1-b), remarkable weak interactions have been observed between $\mathrm{OH}$ group attached to $\mathrm{SO}_{2}$ with adjacent Phenol moiety. 2 virtual bonds $\mathrm{C}_{22}-\mathrm{O}_{33}, \mathrm{C}_{23}-\mathrm{O}_{31}$ of 4.48, $3.12 \AA$, and 9 virtual angles $\mathrm{C}_{21}-\mathrm{C}_{22}-\mathrm{O}_{33}, \mathrm{C}_{24}-\mathrm{C}_{22}-\mathrm{O}_{33}, \mathrm{O}_{33}-\mathrm{C}_{22}-\mathrm{C}_{35}, \mathrm{C}_{21}-\mathrm{C}_{23}-\mathrm{O}_{31}, \mathrm{C}_{25}-\mathrm{C}_{23}-\mathrm{O}_{31}, \mathrm{C}_{26}-\mathrm{C}_{23}-\mathrm{O}_{31}, \mathrm{C}_{23}-\mathrm{O}_{31}-\mathrm{S}_{30}$, $\mathrm{C}_{22}-\mathrm{O}_{33}-\mathrm{S}_{30}, \mathrm{C}_{22}-\mathrm{O}_{33}-\mathrm{H}_{34}$ of $69.01^{\circ}, 88.54^{\circ}, 113.80^{\circ}, 87.71^{\circ}, 124.31^{\circ}, 55.46^{\circ}, 97.45^{\circ}, 100.56^{\circ}$, $59.85^{\circ}$, respectively, registered in Table 1 (see red highlights). Also, 26 virtual torsion angles are registered in Table 1 due to twisted $\mathrm{SO}_{3} \mathrm{H}$-phenol interactions (see red highlights). Such interactions are expected to impact both electronic and photovoltaic properties of m-CP highly.

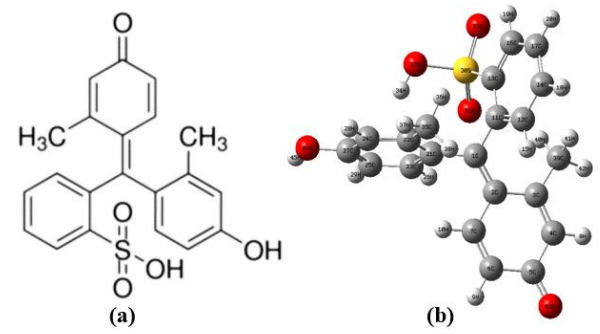

Figure 1. (a) Chemical; (b) Optimum scheme for m-CP. 
Table 1. Calculated m-CP crystal coefficients.

\begin{tabular}{|c|c|c|c|}
\hline bond-extent & $(\AA)$ & torsion-angle & $\left({ }^{\circ}\right)$ \\
\hline $\mathrm{C}_{1}-\mathrm{C}_{2}$ & 1.39 & $\mathrm{C}_{11}-\mathrm{C}_{1}-\mathrm{C}_{2}-\mathrm{C}_{3}$ & -24.75 \\
\hline $\mathrm{C}_{1}-\mathrm{C}_{11}$ & 1.50 & $\mathrm{C}_{11}-\mathrm{C}_{1}-\mathrm{C}_{2}-\mathrm{C}_{7}$ & 155.13 \\
\hline $\mathrm{C}_{1}-\mathrm{C}_{21}$ & 1.50 & $\mathrm{C}_{21}-\mathrm{C}_{1}-\mathrm{C}_{2}-\mathrm{C}_{3}$ & 158.47 \\
\hline $\mathrm{C}_{2}-\mathrm{C}_{3}$ & 1.49 & $\mathrm{C}_{21}-\mathrm{C}_{1}-\mathrm{C}_{2}-\mathrm{C}_{7}$ & -21.65 \\
\hline $\mathrm{C}_{2}-\mathrm{C}_{7}$ & 1.47 & $\mathrm{C}_{2}-\mathrm{C}_{1}-\mathrm{C}_{11}-\mathrm{C}_{12}$ & -56.84 \\
\hline $\mathrm{C}_{3}-\mathrm{C}_{4}$ & 1.37 & $\mathrm{C}_{2}-\mathrm{C}_{1}-\mathrm{C}_{11}-\mathrm{C}_{13}$ & 127.27 \\
\hline $\mathrm{C}_{3}-\mathrm{C}_{39}$ & 1.52 & $\mathrm{C}_{21}-\mathrm{C}_{1}-\mathrm{C}_{11}-\mathrm{C}_{12}$ & 120.05 \\
\hline $\mathrm{C}_{4}-\mathrm{C}_{5}$ & 1.47 & $\mathrm{C}_{21}-\mathrm{C}_{1}-\mathrm{C}_{11}-\mathrm{C}_{13}$ & -55.83 \\
\hline $\mathrm{C}_{4}-\mathrm{H}_{8}$ & 1.09 & $\mathrm{C}_{2}-\mathrm{C}_{1}-\mathrm{C}_{21}-\mathrm{C}_{22}$ & 125.50 \\
\hline $\mathrm{C}_{5}-\mathrm{C}_{6}$ & 1.47 & $\mathrm{C}_{2}-\mathrm{C}_{1}-\mathrm{C}_{21}-\mathrm{C}_{23}$ & -55.59 \\
\hline $\mathrm{C}_{5}-\mathrm{O}_{43}$ & 1.27 & $\mathrm{C}_{11}-\mathrm{C}_{1}-\mathrm{C}_{21}-\mathrm{C}_{22}$ & -51.43 \\
\hline $\mathrm{C}_{6}-\mathrm{C}_{7}$ & 1.36 & $\mathrm{C}_{11}-\mathrm{C}_{1}-\mathrm{C}_{21}-\mathrm{C}_{23}$ & 127.48 \\
\hline $\mathrm{C}_{6}-\mathrm{H}_{9}$ & 1.09 & $\mathrm{C}_{1}-\mathrm{C}_{2}-\mathrm{C}_{3}-\mathrm{C}_{4}$ & 173.92 \\
\hline $\mathrm{C}_{7}-\mathrm{H}_{10}$ & 1.09 & $\mathrm{C}_{1}-\mathrm{C}_{2}-\mathrm{C}_{3}-\mathrm{C}_{39}$ & -13.96 \\
\hline $\mathrm{C}_{11}-\mathrm{C}_{12}$ & 1.42 & $\mathrm{C}_{7}-\mathrm{C}_{2}-\mathrm{C}_{3}-\mathrm{C}_{4}$ & -5.96 \\
\hline $\mathrm{C}_{11}-\mathrm{C}_{13}$ & 1.41 & $\mathrm{C}_{7}-\mathrm{C}_{2}-\mathrm{C}_{3}-\mathrm{C}_{39}$ & 166.16 \\
\hline $\mathrm{C}_{12}-\mathrm{C}_{14}$ & 1.40 & $\mathrm{C}_{1}-\mathrm{C}_{2}-\mathrm{C}_{7}-\mathrm{C}_{6}$ & -172.31 \\
\hline $\mathrm{C}_{12}-\mathrm{H}_{15}$ & 1.09 & $\mathrm{C}_{1}-\mathrm{C}_{2}-\mathrm{C}_{7}-\mathrm{H}_{10}$ & 2.46 \\
\hline $\mathrm{C}_{13}-\mathrm{C}_{16}$ & 1.40 & $\mathrm{C}_{3}-\mathrm{C}_{2}-\mathrm{C}_{7}-\mathrm{C}_{6}$ & 7.58 \\
\hline $\mathrm{C}_{13}-\mathrm{S}_{30}$ & 1.88 & $\mathrm{C}_{3}-\mathrm{C}_{2}-\mathrm{C}_{7}-\mathrm{H}_{10}$ & -177.65 \\
\hline $\mathrm{C}_{14}-\mathrm{C}_{17}$ & 1.41 & $\mathrm{C}_{2}-\mathrm{C}_{3}-\mathrm{C}_{4}-\mathrm{C}_{5}$ & -0.02 \\
\hline $\mathrm{C}_{14}-\mathrm{H}_{18}$ & 1.09 & $\mathrm{C}_{2}-\mathrm{C}_{3}-\mathrm{C}_{4}-\mathrm{H}_{8}$ & 176.98 \\
\hline $\mathrm{C}_{16}-\mathrm{C}_{17}$ & 1.41 & $\mathrm{C}_{39}-\mathrm{C}_{3}-\mathrm{C}_{4}-\mathrm{C}_{5}$ & -172.45 \\
\hline $\mathrm{C}_{16}-\mathrm{H}_{19}$ & 1.09 & $\mathrm{C}_{39}-\mathrm{C}_{3}-\mathrm{C}_{4}-\mathrm{H}_{8}$ & 4.55 \\
\hline $\mathrm{C}_{17}-\mathrm{H}_{20}$ & 1.09 & $\mathrm{C}_{2}-\mathrm{C}_{3}-\mathrm{C}_{39}-\mathrm{C}_{40}$ & -43.22 \\
\hline $\mathrm{C}_{21}-\mathrm{C}_{22}$ & 1.43 & $\mathrm{C}_{2}-\mathrm{C}_{3}-\mathrm{C}_{39}-\mathrm{H}_{41}$ & 78.05 \\
\hline $\mathrm{C}_{21}-\mathrm{C}_{23}$ & 1.42 & $\mathrm{C}_{2}-\mathrm{C}_{3}-\mathrm{C}_{39}-\mathrm{H}_{42}$ & -162.02 \\
\hline $\mathrm{C}_{22}-\mathrm{C}_{24}$ & 1.41 & $\mathrm{C}_{4}-\mathrm{C}_{3}-\mathrm{C}_{39}-\mathrm{H}_{40}$ & 128.96 \\
\hline $\mathrm{C}_{22}-\mathrm{O}_{33}$ & 3.48 & $\mathrm{C}_{4}-\mathrm{C}_{3}-\mathrm{C}_{39}-\mathrm{H}_{41}$ & -109.78 \\
\hline $\mathrm{C}_{22}-\mathrm{C}_{35}$ & 1.52 & $\mathrm{C}_{4}-\mathrm{C}_{3}-\mathrm{C}_{39}-\mathrm{H}_{42}$ & 10.16 \\
\hline $\mathrm{C}_{23}-\mathrm{C}_{25}$ & 1.40 & $\mathrm{C}_{3}-\mathrm{C}_{4}-\mathrm{C}_{5}-\mathrm{C}_{6}$ & 4.74 \\
\hline $\mathrm{C}_{23}-\mathrm{H}_{26}$ & 1.08 & $\mathrm{C}_{3}-\mathrm{C}_{4}-\mathrm{C}_{5}-\mathrm{O}_{43}$ & -176.55 \\
\hline $\mathrm{C}_{23}-\mathrm{O}_{31}$ & 3.12 & $\mathrm{H}_{8}-\mathrm{C}_{4}-\mathrm{C}_{5}-\mathrm{C}_{6}$ & -172.40 \\
\hline $\mathrm{C}_{24}-\mathrm{C}_{27}$ & 1.41 & $\mathrm{H}_{8}-\mathrm{C}_{4}-\mathrm{C}_{5}-\mathrm{O}_{43}$ & 6.31 \\
\hline $\mathrm{C}_{24}-\mathrm{H}_{28}$ & 1.09 & $\mathrm{O}_{33}-\mathrm{C}_{22}-\mathrm{C}_{35}-\mathrm{H}_{36}$ & -4.30 \\
\hline $\mathrm{C}_{25}-\mathrm{C}_{27}$ & 1.41 & $\mathrm{O}_{33}-\mathrm{C}_{22}-\mathrm{C}_{35}-\mathrm{H}_{37}$ & 115.28 \\
\hline $\mathrm{C}_{25}-\mathrm{H}_{29}$ & 1.09 & $\mathrm{O}_{33}-\mathrm{C}_{22}-\mathrm{C}_{35}-\mathrm{H}_{38}$ & -124.47 \\
\hline $\mathrm{C}_{27}-\mathrm{O}_{44}$ & 1.09 & $\mathrm{C}_{21}-\mathrm{C}_{23}-\mathrm{C}_{25}-\mathrm{C}_{27}$ & -1.30 \\
\hline $\mathrm{S}_{30}-\mathrm{O}_{31}$ & 1.61 & $\mathrm{C}_{21}-\mathrm{C}_{23}-\mathrm{C}_{25}-\mathrm{H}_{29}$ & 179.29 \\
\hline $\mathrm{S}_{30}-\mathrm{O}_{32}$ & 1.60 & $\mathrm{H}_{26}-\mathrm{C}_{23}-\mathrm{C}_{25}-\mathrm{C}_{27}$ & -178.70 \\
\hline $\mathrm{S}_{30}-\mathrm{O}_{33}$ & 1.80 & $\mathrm{H}_{29}-\mathrm{C}_{25}-\mathrm{C}_{27}-\mathrm{C}_{24}$ & 179.68 \\
\hline $\mathrm{O}_{33}-\mathrm{H}_{34}$ & 0.99 & $\mathrm{H}_{29}-\mathrm{C}_{25}-\mathrm{C}_{27}-\mathrm{O}_{44}$ & 0.06 \\
\hline $\mathrm{C}_{35}-\mathrm{H}_{36}$ & 1.10 & $\mathrm{C}_{24}-\mathrm{C}_{27}-\mathrm{O}_{44}-\mathrm{H}_{45}$ & -179.30 \\
\hline $\mathrm{C}_{35}-\mathrm{H}_{37}$ & 1.10 & $\mathrm{C}_{25}-\mathrm{C}_{27}-\mathrm{O}_{44}-\mathrm{H}_{45}$ & 0.34 \\
\hline $\mathrm{C}_{35}-\mathrm{H}_{38}$ & 1.10 & $\mathrm{O}_{32}-\mathrm{S}_{30}-\mathrm{O}_{33}-\mathrm{H}_{34}$ & 145.61 \\
\hline $\mathrm{C}_{39}-\mathrm{H}_{40}$ & 1.10 & $\mathrm{C}_{4}-\mathrm{C}_{5}-\mathrm{C}_{6}-\mathrm{C}_{7}$ & -3.23 \\
\hline $\mathrm{C}_{39}-\mathrm{H}_{41}$ & 1.09 & $\mathrm{C}_{4}-\mathrm{C}_{5}-\mathrm{C}_{6}-\mathrm{H}_{9}$ & 178.31 \\
\hline $\mathrm{C}_{39}-\mathrm{H}_{42}$ & 1.09 & $\mathrm{O}_{43}-\mathrm{C}_{5}-\mathrm{C}_{6}-\mathrm{C}_{7}$ & 178.06 \\
\hline $\mathrm{O}_{44}-\mathrm{H}_{45}$ & 0.98 & $\mathrm{O}_{43}-\mathrm{C}_{5}-\mathrm{C}_{6}-\mathrm{H}_{9}$ & -0.40 \\
\hline $\mathrm{C}_{2}-\mathrm{C}_{1}-\mathrm{C}_{11}$ & 122.04 & $\mathrm{C}_{5}-\mathrm{C}_{6}-\mathrm{C}_{7}-\mathrm{C}_{2}$ & -2.90 \\
\hline $\mathrm{C}_{2}-\mathrm{C}_{1}-\mathrm{C}_{21}$ & 120.83 & $\mathrm{C}_{5}-\mathrm{C}_{6}-\mathrm{C}_{7}-\mathrm{H}_{10}$ & -177.56 \\
\hline $\mathrm{C}_{11}-\mathrm{C}_{1}-\mathrm{C}_{21}$ & 117.03 & $\mathrm{H}_{9}-\mathrm{C}_{6}-\mathrm{C}_{7}-\mathrm{C}_{2}$ & 175.48 \\
\hline $\mathrm{C}_{1}-\mathrm{C}_{2}-\mathrm{C}_{3}$ & 125.11 & $\mathrm{H}_{9}-\mathrm{C}_{6}-\mathrm{C}_{7}-\mathrm{H}_{10}$ & 0.82 \\
\hline $\mathrm{C}_{1}-\mathrm{C}_{2}-\mathrm{C}_{7}$ & 118.26 & $\mathrm{C}_{1}-\mathrm{C}_{11}-\mathrm{C}_{12}-\mathrm{C}_{14}$ & -175.00 \\
\hline $\mathrm{C}_{3}-\mathrm{C}_{2}-\mathrm{C}_{7}$ & 116.63 & $\mathrm{C}_{1}-\mathrm{C}_{11}-\mathrm{C}_{12}-\mathrm{H}_{15}$ & 3.69 \\
\hline $\mathrm{C}_{2}-\mathrm{C}_{3}-\mathrm{C}_{4}$ & 119.00 & $\mathrm{C}_{13}-\mathrm{C}_{11}-\mathrm{C}_{12}-\mathrm{C}_{14}$ & 1.35 \\
\hline $\mathrm{C}_{2}-\mathrm{C}_{3}-\mathrm{C}_{39}$ & 122.21 & $\mathrm{C}_{13}-\mathrm{C}_{11}-\mathrm{C}_{12}-\mathrm{H}_{15}$ & -179.95 \\
\hline $\mathrm{C}_{4}-\mathrm{C}_{3}-\mathrm{C}_{39}$ & 118.34 & $\mathrm{C}_{1}-\mathrm{C}_{11}-\mathrm{C}_{13}-\mathrm{C}_{16}$ & 174.61 \\
\hline $\mathrm{C}_{3}-\mathrm{C}_{4}-\mathrm{C}_{5}$ & 124.10 & $\mathrm{C}_{1}-\mathrm{C}_{11}-\mathrm{C}_{13}-\mathrm{S}_{30}$ & -12.33 \\
\hline $\mathrm{C}_{3}-\mathrm{C}_{4}-\mathrm{H}_{8}$ & 120.49 & $\mathrm{C}_{12}-\mathrm{C}_{11}-\mathrm{C}_{13}-\mathrm{C}_{16}$ & -1.37 \\
\hline $\mathrm{C}_{5}-\mathrm{C}_{4}-\mathrm{H}_{8}$ & 115.35 & $\mathrm{C}_{12}-\mathrm{C}_{11}-\mathrm{C}_{13}-\mathrm{S}_{30}$ & 171.69 \\
\hline $\mathrm{C}_{4}-\mathrm{C}_{5}-\mathrm{C}_{6}$ & 115.88 & $\mathrm{C}_{11}-\mathrm{C}_{12}-\mathrm{C}_{14}-\mathrm{C}_{17}$ & -0.14 \\
\hline $\mathrm{C}_{4}-\mathrm{C}_{5}-\mathrm{O}_{43}$ & 121.90 & $\mathrm{C}_{11}-\mathrm{C}_{12}-\mathrm{C}_{14}-\mathrm{H}_{18}$ & 179.71 \\
\hline $\mathrm{C}_{6}-\mathrm{C}_{5}-\mathrm{O}_{43}$ & 122.20 & $\mathrm{H}_{15}-\mathrm{C}_{12}-\mathrm{C}_{14}-\mathrm{C}_{17}$ & -178.81 \\
\hline $\mathrm{C}_{5}-\mathrm{C}_{6}-\mathrm{C}_{7}$ & 120.85 & $\mathrm{H}_{15}-\mathrm{C}_{12}-\mathrm{C}_{14}-\mathrm{H}_{18}$ & 1.04 \\
\hline
\end{tabular}




\begin{tabular}{|c|c|c|c|}
\hline bond-extent & $(\AA)$ & torsion-angle & $\left({ }^{\circ}\right)$ \\
\hline $\mathrm{C}_{5}-\mathrm{C}_{6}-\mathrm{H}_{9}$ & 117.14 & $\mathrm{H}_{11}-\mathrm{C}_{13}-\mathrm{C}_{16}-\mathrm{C}_{17}$ & 0.14 \\
\hline $\mathrm{C}_{7}-\mathrm{C}_{6}-\mathrm{H}_{9}$ & 121.99 & $\mathrm{H}_{11}-\mathrm{C}_{13}-\mathrm{C}_{16}-\mathrm{H}_{19}$ & 178.12 \\
\hline $\mathrm{C}_{2}-\mathrm{C}_{7}-\mathrm{C}_{6}$ & 122.97 & $\mathrm{~S}_{30}-\mathrm{C}_{13}-\mathrm{C}_{16}-\mathrm{C}_{17}$ & -173.45 \\
\hline $\mathrm{C}_{2}-\mathrm{C}_{7}-\mathrm{H}_{10}$ & 117.42 & $\mathrm{~S}_{30}-\mathrm{C}_{13}-\mathrm{C}_{16}-\mathrm{H}_{19}$ & 4.54 \\
\hline $\mathrm{C}_{6}-\mathrm{C}_{7}-\mathrm{H}_{10}$ & 119.42 & $\mathrm{C}_{11}-\mathrm{C}_{13}-\mathrm{S}_{30}-\mathrm{O}_{31}$ & -30.28 \\
\hline $\mathrm{C}_{1}-\mathrm{C}_{11}-\mathrm{C}_{12}$ & 117.75 & $\mathrm{C}_{11}-\mathrm{C}_{13}-\mathrm{S}_{30}-\mathrm{O}_{32}$ & -163.21 \\
\hline $\mathrm{C}_{1}-\mathrm{C}_{11}-\mathrm{C}_{13}$ & 126.91 & $\mathrm{C}_{11}-\mathrm{C}_{13}-\mathrm{S}_{30}-\mathrm{O}_{33}$ & 86.29 \\
\hline $\mathrm{C}_{12}-\mathrm{C}_{11}-\mathrm{C}_{13}$ & 115.22 & $\mathrm{C}_{16}-\mathrm{C}_{13}-\mathrm{S}_{30}-\mathrm{O}_{31}$ & 143.47 \\
\hline $\mathrm{C}_{11}-\mathrm{C}_{12}-\mathrm{C}_{14}$ & 122.09 & $\mathrm{C}_{16}-\mathrm{C}_{13}-\mathrm{S}_{30}-\mathrm{O}_{32}$ & 10.54 \\
\hline $\mathrm{C}_{11}-\mathrm{C}_{12}-\mathrm{C}_{15}$ & 118.00 & $\mathrm{C}_{16}-\mathrm{C}_{13}-\mathrm{S}_{30}-\mathrm{O}_{33}$ & -99.96 \\
\hline $\mathrm{C}_{14}-\mathrm{C}_{12}-\mathrm{C}_{15}$ & 119.90 & $\mathrm{C}_{12}-\mathrm{C}_{14}-\mathrm{C}_{17}-\mathrm{C}_{16}$ & -1.17 \\
\hline $\mathrm{C}_{11}-\mathrm{C}_{13}-\mathrm{C}_{16}$ & 124.21 & $\mathrm{C}_{12}-\mathrm{C}_{14}-\mathrm{C}_{17}-\mathrm{H}_{20}$ & 179.76 \\
\hline $\mathrm{C}_{11}-\mathrm{C}_{13}-\mathrm{S}_{30}$ & 122.06 & $\mathrm{H}_{18}-\mathrm{C}_{14}-\mathrm{C}_{17}-\mathrm{C}_{16}$ & 178.98 \\
\hline $\mathrm{C}_{16}-\mathrm{C}_{13}-\mathrm{S}_{30}$ & 113.41 & $\mathrm{H}_{18}-\mathrm{C}_{14}-\mathrm{C}_{17}-\mathrm{H}_{20}$ & -0.09 \\
\hline $\mathrm{C}_{12}-\mathrm{C}_{14}-\mathrm{C}_{17}$ & 120.42 & $\mathrm{H}_{26}-\mathrm{C}_{23}-\mathrm{C}_{25}-\mathrm{H}_{29}$ & 1.89 \\
\hline $\mathrm{C}_{12}-\mathrm{C}_{14}-\mathrm{C}_{18}$ & 119.50 & $\mathrm{O}_{31}-\mathrm{C}_{23}-\mathrm{C}_{25}-\mathrm{C}_{27}$ & -112.17 \\
\hline $\mathrm{C}_{17}-\mathrm{C}_{14}-\mathrm{C}_{18}$ & 120.08 & $\mathrm{O}_{31}-\mathrm{C}_{23}-\mathrm{C}_{25}-\mathrm{H}_{29}$ & 68.43 \\
\hline $\mathrm{C}_{13}-\mathrm{C}_{16}-\mathrm{C}_{17}$ & 118.72 & $\mathrm{C}_{21}-\mathrm{C}_{23}-\mathrm{O}_{31}-\mathrm{S}_{30}$ & -47.29 \\
\hline $\mathrm{C}_{13}-\mathrm{C}_{16}-\mathrm{H}_{19}$ & 119.82 & $\mathrm{C}_{25}-\mathrm{C}_{23}-\mathrm{O}_{31}-\mathrm{S}_{30}$ & 80.21 \\
\hline $\mathrm{C}_{17}-\mathrm{C}_{16}-\mathrm{H}_{19}$ & 121.43 & $\mathrm{H}_{26}-\mathrm{C}_{23}-\mathrm{O}_{31}-\mathrm{S}_{30}$ & -175.10 \\
\hline $\mathrm{C}_{14}-\mathrm{C}_{17}-\mathrm{C}_{16}$ & 119.32 & $\mathrm{C}_{13}-\mathrm{S}_{30}-\mathrm{O}_{31}-\mathrm{C}_{23}$ & 74.02 \\
\hline $\mathrm{C}_{14}-\mathrm{C}_{17}-\mathrm{H}_{20}$ & 120.78 & $\mathrm{O}_{32}-\mathrm{S}_{30}-\mathrm{O}_{31}-\mathrm{C}_{23}$ & -159.91 \\
\hline $\mathrm{C}_{16}-\mathrm{C}_{17}-\mathrm{H}_{20}$ & 119.89 & $\mathrm{O}_{33}-\mathrm{S}_{30}-\mathrm{O}_{31}-\mathrm{C}_{23}$ & -39.44 \\
\hline $\mathrm{C}_{1}-\mathrm{C}_{21}-\mathrm{C}_{22}$ & 123.86 & $\mathrm{C}_{13-}-\mathrm{S}_{30}-\mathrm{O}_{31}-\mathrm{C}_{22}$ & -41.42 \\
\hline $\mathrm{C}_{1}-\mathrm{C}_{21}-\mathrm{C}_{23}$ & 116.99 & $\mathrm{C}_{13}-\mathrm{C}_{16}-\mathrm{C}_{17}-\mathrm{C}_{14}$ & 1.16 \\
\hline $\mathrm{C}_{22}-\mathrm{C}_{21}-\mathrm{C}_{23}$ & 119.14 & $\mathrm{C}_{13}-\mathrm{C}_{16}-\mathrm{C}_{17}-\mathrm{H}_{20}$ & -179.76 \\
\hline $\mathrm{C}_{21}-\mathrm{C}_{22}-\mathrm{C}_{24}$ & 118.49 & $\mathrm{H}_{19}-\mathrm{C}_{16}-\mathrm{C}_{17}-\mathrm{C}_{14}$ & -176.79 \\
\hline $\mathrm{C}_{21}-\mathrm{C}_{22}-\mathrm{O}_{33}$ & 69.01 & $\mathrm{H}_{19}-\mathrm{C}_{16}-\mathrm{C}_{17}-\mathrm{H}_{20}$ & 2.29 \\
\hline $\mathrm{C}_{21}-\mathrm{C}_{22}-\mathrm{C}_{35}$ & 122.93 & $\mathrm{C}_{1}-\mathrm{C}_{21}-\mathrm{C}_{22}-\mathrm{C}_{24}$ & 178.52 \\
\hline $\mathrm{C}_{24}-\mathrm{C}_{22}-\mathrm{O}_{33}$ & 88.54 & $\mathrm{C}_{1}-\mathrm{C}_{21}-\mathrm{C}_{22}-\mathrm{O}_{33}$ & 101.34 \\
\hline $\mathrm{C}_{24}-\mathrm{C}_{22}-\mathrm{C}_{35}$ & 118.56 & $\mathrm{C}_{1}-\mathrm{C}_{21}-\mathrm{C}_{22}-\mathrm{C}_{35}$ & -3.35 \\
\hline $\mathrm{O}_{33}-\mathrm{C}_{22}-\mathrm{C}_{35}$ & 113.80 & $\mathrm{C}_{23}-\mathrm{C}_{21}-\mathrm{C}_{22}-\mathrm{C}_{24}$ & -0.38 \\
\hline $\mathrm{C}_{21}-\mathrm{C}_{23}-\mathrm{C}_{25}$ & 121.45 & $\mathrm{C}_{23}-\mathrm{C}_{21}-\mathrm{C}_{22}-\mathrm{O}_{33}$ & -77.56 \\
\hline $\mathrm{C}_{21}-\mathrm{C}_{23}-\mathrm{C}_{26}$ & 118.55 & $\mathrm{C}_{23}-\mathrm{C}_{21}-\mathrm{C}_{22}-\mathrm{C}_{35}$ & 177.76 \\
\hline $\mathrm{C}_{21}-\mathrm{C}_{23}-\mathrm{O}_{31}$ & 87.71 & $\mathrm{C}_{1}-\mathrm{C}_{21}-\mathrm{C}_{23}-\mathrm{C}_{25}$ & -177.62 \\
\hline $\mathrm{C}_{25}-\mathrm{C}_{23}-\mathrm{C}_{26}$ & 119.96 & $\mathrm{C}_{1}-\mathrm{C}_{21}-\mathrm{C}_{23}-\mathrm{H}_{26}$ & -0.20 \\
\hline $\mathrm{C}_{25}-\mathrm{C}_{23}-\mathrm{O}_{31}$ & 124.31 & $\mathrm{C}_{1}-\mathrm{C}_{21}-\mathrm{C}_{23}-\mathrm{O}_{31}$ & -48.23 \\
\hline $\mathrm{C}_{26}-\mathrm{C}_{23}-\mathrm{O}_{31}$ & 55.46 & $\mathrm{C}_{22}-\mathrm{C}_{21}-\mathrm{C}_{23}-\mathrm{C}_{25}$ & 1.35 \\
\hline $\mathrm{C}_{22}-\mathrm{C}_{24}-\mathrm{C}_{27}$ & 121.95 & $\mathrm{C}_{22}-\mathrm{C}_{21}-\mathrm{C}_{23}-\mathrm{H}_{26}$ & 178.78 \\
\hline $\mathrm{C}_{22}-\mathrm{C}_{24}-\mathrm{C}_{28}$ & 118.72 & $\mathrm{C}_{22}-\mathrm{C}_{21}-\mathrm{C}_{23}-\mathrm{O}_{31}$ & 130.74 \\
\hline $\mathrm{C}_{27}-\mathrm{C}_{24}-\mathrm{C}_{28}$ & 119.33 & $\mathrm{C}_{21}-\mathrm{C}_{22}-\mathrm{C}_{24}-\mathrm{C}_{27}$ & -0.61 \\
\hline $\mathrm{C}_{23}-\mathrm{C}_{25}-\mathrm{C}_{27}$ & 119.42 & $\mathrm{C}_{21}-\mathrm{C}_{22}-\mathrm{C}_{24}-\mathrm{H}_{28}$ & 179.07 \\
\hline $\mathrm{C}_{23}-\mathrm{C}_{25}-\mathrm{H}_{29}$ & 119.95 & $\mathrm{O}_{33}-\mathrm{C}_{22}-\mathrm{C}_{24}-\mathrm{C}_{27}$ & 65.24 \\
\hline $\mathrm{C}_{27}-\mathrm{C}_{25}-\mathrm{H}_{29}$ & 120.64 & $\mathrm{O}_{33}-\mathrm{C}_{22}-\mathrm{C}_{24}-\mathrm{H}_{28}$ & -115.08 \\
\hline $\mathrm{C}_{24}-\mathrm{C}_{27}-\mathrm{C}_{25}$ & 119.54 & $\mathrm{C}_{35}-\mathrm{C}_{22}-\mathrm{C}_{24}-\mathrm{C}_{27}$ & -178.83 \\
\hline $\mathrm{C}_{24}-\mathrm{C}_{27}-\mathrm{O}_{44}$ & 120.05 & $\mathrm{C}_{35}-\mathrm{C}_{22}-\mathrm{C}_{24}-\mathrm{H}_{28}$ & 0.85 \\
\hline $\mathrm{C}_{25}-\mathrm{C}_{27}-\mathrm{O}_{44}$ & 120.41 & $\mathrm{C}_{21}-\mathrm{C}_{22}-\mathrm{O}_{33}-\mathrm{S}_{30}$ & -40.03 \\
\hline $\mathrm{C}_{13}-\mathrm{S}_{30}-\mathrm{O}_{31}$ & 110.10 & $\mathrm{C}_{21}-\mathrm{C}_{22}-\mathrm{O}_{33}-\mathrm{H}_{34}$ & 66.12 \\
\hline $\mathrm{C}_{13}-\mathrm{S}_{30}-\mathrm{O}_{32}$ & 107.56 & $\mathrm{C}_{24}-\mathrm{C}_{22}-\mathrm{O}_{33}-\mathrm{S}_{30}$ & -161.55 \\
\hline $\mathrm{C}_{13}-\mathrm{S}_{30}-\mathrm{O}_{33}$ & 103.80 & $\mathrm{C}_{24}-\mathrm{C}_{22}-\mathrm{O}_{33}-\mathrm{H}_{34}$ & -55.41 \\
\hline $\mathrm{O}_{31}-\mathrm{S}_{30}-\mathrm{O}_{32}$ & 120.35 & $\mathrm{C}_{35}-\mathrm{C}_{22}-\mathrm{O}_{33}-\mathrm{S}_{30}$ & 77.92 \\
\hline $\mathrm{O}_{31}-\mathrm{S}_{30}-\mathrm{O}_{33}$ & 109.09 & $\mathrm{C}_{35}-\mathrm{C}_{22}-\mathrm{O}_{33}-\mathrm{H}_{34}$ & -175.94 \\
\hline $\mathrm{O}_{32}-\mathrm{S}_{30}-\mathrm{O}_{33}$ & 104.62 & $\mathrm{C}_{21}-\mathrm{C}_{22}-\mathrm{C}_{35}-\mathrm{H}_{36}$ & 75.18 \\
\hline $\mathrm{C}_{23}-\mathrm{O}_{31}-\mathrm{S}_{30}$ & 97.45 & $\mathrm{C}_{21}-\mathrm{C}_{22}-\mathrm{C}_{35}-\mathrm{H}_{37}$ & -165.24 \\
\hline $\mathrm{C}_{22}-\mathrm{O}_{33}-\mathrm{S}_{30}$ & 100.56 & $\mathrm{C}_{21}-\mathrm{C}_{22}-\mathrm{C}_{35}-\mathrm{H}_{38}$ & -44.99 \\
\hline $\mathrm{C}_{22}-\mathrm{O}_{33}-\mathrm{H}_{34}$ & 59.85 & $\mathrm{C}_{24}-\mathrm{C}_{22}-\mathrm{C}_{35}-\mathrm{H}_{36}$ & -106.67 \\
\hline $\mathrm{S}_{30}-\mathrm{O}_{33}-\mathrm{H}_{34}$ & 109.38 & $\mathrm{C}_{24}-\mathrm{C}_{22}-\mathrm{C}_{35}-\mathrm{H}_{37}$ & 12.91 \\
\hline $\mathrm{C}_{22}-\mathrm{C}_{35}-\mathrm{H}_{36}$ & 111.65 & $\mathrm{C}_{24}-\mathrm{C}_{22}-\mathrm{C}_{35}-\mathrm{H}_{38}$ & 133.16 \\
\hline $\mathrm{C}_{22}-\mathrm{C}_{35}-\mathrm{H}_{37}$ & 110.45 & $\mathrm{C}_{22}-\mathrm{C}_{24}-\mathrm{C}_{27}-\mathrm{C}_{25}$ & 0.67 \\
\hline $\mathrm{C}_{22}-\mathrm{C}_{35}-\mathrm{H}_{38}$ & 111.86 & $\mathrm{C}_{22}-\mathrm{C}_{24}-\mathrm{C}_{27}-\mathrm{O}_{44}$ & -179.68 \\
\hline $\mathrm{H}_{36}-\mathrm{C}_{35}-\mathrm{H}_{37}$ & 107.47 & $\mathrm{H}_{28}-\mathrm{C}_{24}-\mathrm{C}_{27}-\mathrm{C}_{25}$ & -179.01 \\
\hline $\mathrm{H}_{36}-\mathrm{C}_{35}-\mathrm{H}_{38}$ & 107.37 & $\mathrm{H}_{28}-\mathrm{C}_{24}-\mathrm{C}_{27}-\mathrm{O}_{44}$ & 0.63 \\
\hline $\mathrm{H}_{37}-\mathrm{C}_{35}-\mathrm{H}_{38}$ & 107.84 & $\mathrm{C}_{23}-\mathrm{C}_{25}-\mathrm{C}_{27}-\mathrm{C}_{24}$ & 0.28 \\
\hline $\mathrm{C}_{3}-\mathrm{C}_{39}-\mathrm{H}_{40}$ & 110.69 & $\mathrm{C}_{23}-\mathrm{C}_{25}-\mathrm{C}_{27}-\mathrm{O}_{44}$ & -179.35 \\
\hline $\mathrm{C}_{3}-\mathrm{C}_{39}-\mathrm{H}_{41}$ & 113.18 & $\mathrm{C}_{13}-\mathrm{S}_{30}-\mathrm{O}_{33}-\mathrm{H}_{34}$ & -101.68 \\
\hline $\mathrm{C}_{3}-\mathrm{C}_{39}-\mathrm{H}_{42}$ & 109.51 & $\mathrm{O}_{31}-\mathrm{S}_{30}-\mathrm{O}_{33}-\mathrm{C}_{22}$ & 76.12 \\
\hline
\end{tabular}




\begin{tabular}{c|c|c|c} 
bond-extent & $(\AA)$ & torsion-angle & $\left({ }^{\circ}\right)$ \\
\hline $\mathrm{H}_{40}-\mathrm{C}_{39}-\mathrm{H}_{41}$ & 107.91 & $\mathrm{O}_{31}-\mathrm{S}_{30}-\mathrm{O}_{33}-\mathrm{H}_{34}$ & 15.86 \\
\hline $\mathrm{H}_{40}-\mathrm{C}_{39}-\mathrm{H}_{42}$ & 107.85 & $\mathrm{O}_{32}-\mathrm{S}_{30}-\mathrm{O}_{33}-\mathrm{C}_{22}$ & -154.13 \\
\hline $\mathrm{H}_{41}-\mathrm{C}_{39}-\mathrm{H}_{42}$ & 107.52 & & \\
\hline $\mathrm{C}_{27}-\mathrm{O}_{44}-\mathrm{H}_{45}$ & 107.95 & &
\end{tabular}

\subsection{FTIR analyses.}

For convenience, authors select only 27 modes among 129 others to concur with recorded m-CP FTIR wavenumbers assigned in Table 2. Assignments are performed through GV 5, considering that the predicted wavenumber is a scale of 0.96 [31] to fit with experimental data. A proper harmony between measured and predicted FTIR charts is noticed. Fig. 2 shows both measured and computed FTIR charts. m-CP assignments are briefly discussed as:

\subsubsection{Single-bond vibrations.}

$\mathrm{OH}$ stretch is detected between $3400-3500 \mathrm{~cm}^{-1}$ [32]. Mode 1 is assigned to $\mathrm{OH}$ stretch at $3409 \mathrm{~cm}^{-1}$, comparable to experimental at $3450 \mathrm{~cm}^{-1}$. Mode 12 is attributed to $\mathrm{OH}$ scissoring at $1243 \mathrm{~cm}^{-1}$, commensurate to an observed band at $1246 \mathrm{~cm}^{-1}$. Modes $(18,19)$ are referred to $\mathrm{OH}$ wagging and twisting at $884,852 \mathrm{~cm}^{-1}$, balanced to recorded bands at $885,850 \mathrm{~cm}^{-1}$, respectively.

$\mathrm{CH}$ stretch is perceived among $3300-3000 \mathrm{~cm}^{-1}$ [33]. Modes $(2,3)$ are imputed to both asymmetric and symmetric terminal $\mathrm{CH}$ stretch at $3116,3114 \mathrm{~cm}^{-1}$, corresponding to experimental ones at $3242,3160 \mathrm{~cm}^{-1}$. Modes $(4,5)$ are referred to as both non-symmetric and symmetric ring $\mathrm{CH}$ stretch at $3100,3085 \mathrm{~cm}^{-1}$, analogous to recorded bands at 3100,3050 $\mathrm{cm}^{-1}$. Modes (13-15) are specified for $\mathrm{CH}$ scissoring and rocking at $1226,1131,1068 \mathrm{~cm}^{-1}$, proportionate to measured values at $1215,1122,1083 \mathrm{~cm}^{-1}$, respectively. Modes (19-22) are related to $\mathrm{CH}$ wagging and twisting at $852,795,765,729 \mathrm{~cm}^{-1}$, analogous to recognized peaks at $850,772,760,723 \mathrm{~cm}^{-1}$, respectively. Modes $(10,11)$ are mentioned to $\mathrm{CH}_{3}$ deformations at $1470,1434 \mathrm{~cm}^{-1}$, analogous to indexed data at $1467,1433 \mathrm{~cm}^{-1}$.

Mode 16 is attributed to SO+SC stretch at $1025 \mathrm{~cm}^{-1}$, analogous to defined peak at $1016 \mathrm{~cm}^{-1}$ [34]. Mode 17 is allocated to CO+CC stretch at $996 \mathrm{~cm}^{-1}$, comparable to the observed value at $960 \mathrm{~cm}^{-1}$. Mode 23 is specified for $\mathrm{SO}+\mathrm{SC}+\mathrm{CO}+\mathrm{CC}$ scissoring at $671 \mathrm{~cm}^{-1}$, corresponding to index measure at $666 \mathrm{~cm}^{-1}$. Mode 24 is verified for $\mathrm{SO}+\mathrm{SC}+\mathrm{CO}+\mathrm{CC}$ wagging at $567 \mathrm{~cm}^{-1}$, comparative to the recorded pattern at $556 \mathrm{~cm}^{-1}$.

\subsubsection{Double-bond vibrations.}

$\mathrm{C}=\mathrm{O}$, as well as $\mathrm{C}=\mathrm{C}$ stretch, is noticed amidst $1750-1500 \mathrm{~cm}^{-1}$ [35]. Mode 6 is approved for $\mathrm{C}=\mathrm{O}$ stretch at $1612 \mathrm{~cm}^{-1}$, correspond to the observed value at $1613 \mathrm{~cm}^{-1}$. Modes (7-9) are referred to $\mathrm{C}=\mathrm{C}$ stretch at $1593,1542,1540 \mathrm{~cm}^{-1}$, concur to experimental data at $1583,1552,1506 \mathrm{~cm}^{-1}$.

\subsection{Mulliken distribution analyses.}

A direct way to describe molecule electronic properties such as free carriers transport or electro negatively can be easily achieved through Mulliken distribution of charges [36]. Mulliken distributions of m-CP partial charges (Q) are registered in Table 3. Distribution of Mulliken m-CP partial charges present in Fig. 3. Positive Q-values are allocated on $C_{1}, C_{2}, C_{3}$, 
$\mathrm{C}_{5}, \mathrm{C}_{10}, \mathrm{C}_{11}, \mathrm{C}_{21}, \mathrm{C}_{22}$, and all $\mathrm{H}$ atoms, while negative $\mathrm{Q}$-values are set on others. Unusual electrostatic interactions are observed between $\mathrm{O}_{33}$ with both $\mathrm{C}_{22}$ and $\mathrm{C}_{23}$, which are expected to impact NLO behavior of $\mathrm{m}-\mathrm{CP}$.

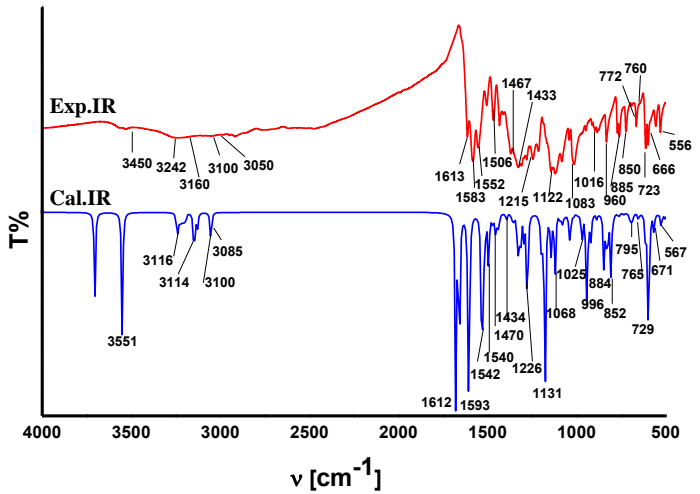

Figure 2. Observed and calculated FTIR charts for m-CP.

Table 2. Recorded and theoretical FTIR wavenumbers, IR intensities and corresponding assignments for m-CP.

\begin{tabular}{|c|c|c|c|c|c|c|}
\hline \multirow{2}{*}{ No } & \multirow{2}{*}{$\operatorname{Exp} .\left(\mathrm{cm}^{-1}\right)$} & \multicolumn{2}{|c|}{ Cal. $\left(\mathrm{cm}^{-1}\right)$} & \multicolumn{2}{|c|}{ IR } & \multirow{2}{*}{ Vibrational assignments } \\
\hline & & Non-scaled & Scaled & Rel. & Abs. & \\
\hline 1 & 3450 & 3551 & 3409 & 127 & 60 & $v \mathrm{OH}$ \\
\hline 2 & 3242 & 3246 & 3116 & 0 & 0 & $\mathrm{v}_{\mathrm{as}} \mathrm{CH}\left(\mathrm{CH}_{3}\right)$ \\
\hline 3 & 3160 & 3244 & 3114 & 15 & 7 & $v_{\mathrm{s}} \mathrm{CH}\left(\mathrm{CH}_{3}\right)$ \\
\hline 4 & 3100 & 3229 & 3100 & 6 & 3 & vas CH (Ring) \\
\hline 5 & 3050 & 3214 & 3085 & 10 & 5 & $v_{\mathrm{s}} \mathrm{CH}$ (Ring) \\
\hline 6 & 1613 & 1679 & 1612 & 200 & 94 & v $\mathrm{C}=\mathrm{O}$ \\
\hline 7 & 1583 & 1659 & 1593 & 171 & 80 & \multirow{3}{*}{ v $\mathrm{C}=\mathrm{C}$} \\
\hline 8 & 1552 & 1606 & 1542 & 109 & 51 & \\
\hline 9 & 1506 & 1604 & 1540 & 124 & 58 & \\
\hline 10 & 1467 & 1531 & 1470 & 119 & 56 & \multirow{2}{*}{$\mathrm{CH}_{3}$ deformations } \\
\hline 11 & 1433 & 1494 & 1434 & 57 & 27 & \\
\hline 12 & 1246 & 1295 & 1243 & 24 & 11 & $\zeta \mathrm{OH}$ \\
\hline 13 & 1215 & 1277 & 1226 & 107 & 50 & $\zeta \mathrm{CH}$ \\
\hline 14 & 1122 & 1178 & 1131 & 213 & 100 & $\zeta \mathrm{CH}$ \\
\hline 15 & 1083 & 1112 & 1068 & 45 & 21 & $\rho \mathrm{CH}$ \\
\hline 16 & 1016 & 1068 & 1025 & 5 & 2 & $v \mathrm{SO}+\mathrm{SC}$ \\
\hline 17 & 960 & 1037 & 996 & 35 & 16 & v $\mathrm{CO}+\mathrm{CC}$ \\
\hline 18 & 885 & 921 & 884 & 20 & 9 & $\tau \mathrm{OH}$ \\
\hline 19 & 850 & 887 & 852 & 6 & 3 & $\omega \mathrm{OH}$ \\
\hline 20 & 772 & 828 & 795 & 128 & 60 & $\tau \mathrm{CH}$ \\
\hline 21 & 760 & 797 & 765 & 10 & 5 & $\tau \mathrm{CH}$ \\
\hline 22 & 723 & 759 & 729 & 2 & 1 & $\omega \mathrm{CH}$ \\
\hline 23 & 666 & 699 & 671 & 14 & 7 & $\zeta \mathrm{SO}+\mathrm{SC}+\mathrm{CO}+\mathrm{CC}$ \\
\hline 24 & 556 & 591 & 567 & 35 & 16 & $\omega \mathrm{SO}+\mathrm{SC}+\mathrm{CO}+\mathrm{CC}$ \\
\hline
\end{tabular}

$v$ (stretch); $v_{s}$ (symmetric); $v_{\text {as }}$ (non - symmetric); $\zeta$ (scissoring); $\rho$ (rocking); $\omega$ (wagging); $\tau$ (twisting)

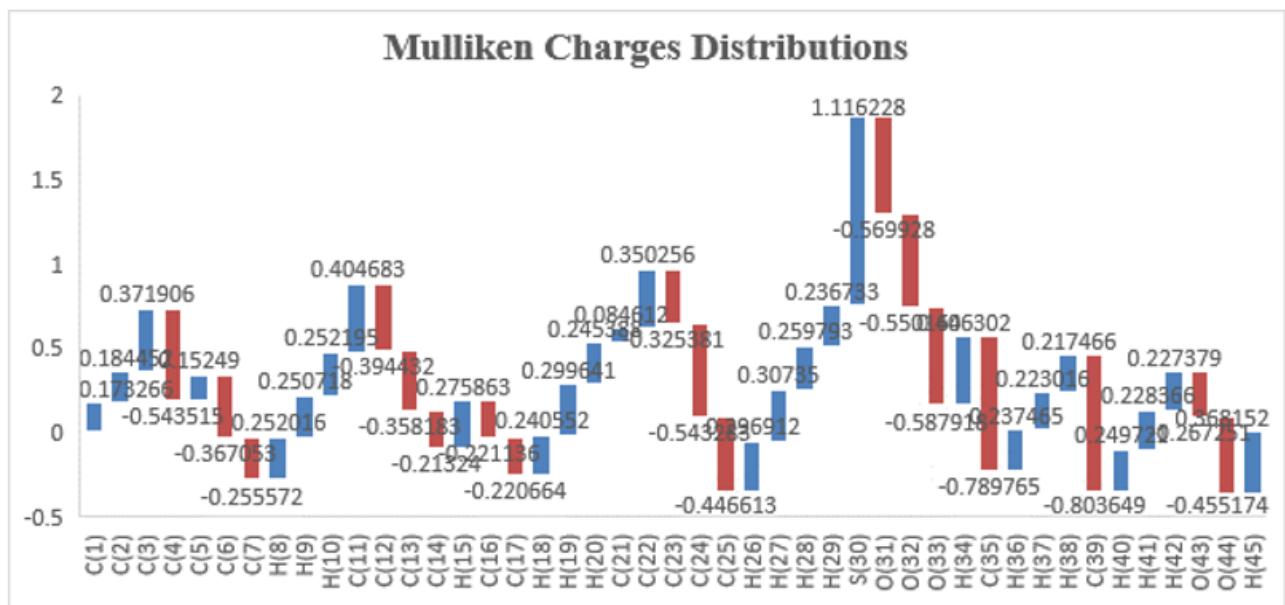

Figure 3. Mulliken distributions for $\mathrm{m}-\mathrm{CP}$ charges. 
Table 3. Mulliken distributions for $\mathrm{m}-\mathrm{CP}$ charges.

\begin{tabular}{|c|c|c|c|}
\hline Atom & $\mathbf{Q}(\mathbf{a . u})$ & Atom & $\mathbf{Q}(\mathbf{a . u})$ \\
\hline $\mathrm{C}_{1}$ & 0.173266 & $\mathrm{C}_{24}$ & -0.543283 \\
\hline $\mathrm{C}_{2}$ & 0.184452 & $\mathrm{C}_{25}$ & -0.446613 \\
\hline $\mathrm{C}_{3}$ & 0.371906 & $\mathrm{H}_{26}$ & 0.296912 \\
\hline $\mathrm{C}_{4}$ & -0.543515 & $\mathrm{H}_{27}$ & 0.30735 \\
\hline $\mathrm{C}_{5}$ & 0.15249 & $\mathrm{H}_{28}$ & 0.259793 \\
\hline $\mathrm{C}_{6}$ & -0.367053 & $\mathrm{H}_{29}$ & 0.236733 \\
\hline $\mathrm{C}_{7}$ & -0.255572 & $\mathrm{~S}_{30}$ & 1.116228 \\
\hline $\mathrm{H}_{8}$ & 0.252016 & $\mathrm{O}_{31}$ & -0.569928 \\
\hline $\mathrm{H}_{9}$ & 0.250718 & $\mathrm{O}_{32}$ & -0.550164 \\
\hline $\mathrm{H}_{10}$ & 0.252195 & $\mathrm{O}_{33}$ & -0.587918 \\
\hline $\mathrm{C}_{11}$ & 0.404683 & $\mathrm{H}_{34}$ & 0.406302 \\
\hline $\mathrm{C}_{12}$ & -0.394432 & $\mathrm{C}_{35}$ & -0.789765 \\
\hline $\mathrm{C}_{13}$ & -0.358183 & $\mathrm{H}_{36}$ & 0.237465 \\
\hline $\mathrm{C}_{14}$ & -0.21324 & $\mathrm{H}_{37}$ & 0.223016 \\
\hline $\mathrm{H}_{15}$ & 0.275863 & $\mathrm{H}_{38}$ & 0.217466 \\
\hline $\mathrm{C}_{16}$ & -0.221136 & $\mathrm{C}_{39}$ & -0.803649 \\
\hline $\mathrm{C}_{17}$ & -0.220664 & $\mathrm{H}_{40}$ & 0.249721 \\
\hline $\mathrm{H}_{18}$ & 0.240552 & $\mathrm{H}_{41}$ & 0.228366 \\
\hline $\mathrm{H}_{19}$ & 0.299641 & $\mathrm{H}_{42}$ & 0.227379 \\
\hline $\mathrm{H}_{20}$ & 0.245388 & $\mathrm{O}_{43}$ & -0.267251 \\
\hline $\mathrm{C}_{21}$ & 0.084612 & $\mathrm{O}_{44}$ & -0.455174 \\
\hline $\mathrm{C}_{22}$ & 0.350256 & $\mathrm{H}_{45}$ & 0.368152 \\
\hline $\mathrm{C}_{23}$ & -0.325381 & & \\
\hline
\end{tabular}

\subsection{Thermo-chemistry and frontier molecular orbital (FMOs) analyses.}

Thermo-dynamical parameters like total-energy (TE), absolute zero-energy (AZE), rotation-coefficients $(\mathrm{RCs})$, entropy $(\mathrm{S})$, specific-heat $(\mathrm{Cv})$, overall dipole-moment (TDM), nuclear repulsive-potential (NRP), and HOMO LUMO offset for $\mathrm{m}-\mathrm{CP}$ are calculated and listed in Table 4.

Table 4 Thermo-chemistry, $\mathrm{E}_{\mathrm{Lumo}}, \mathrm{E}_{\mathrm{HOMO}}$ and $\mathrm{E}_{\mathrm{LUMO} / \mathrm{HOMO}}$ offset for $\mathrm{m}-\mathrm{CP}$ before and after UV exposure.

\begin{tabular}{|c|c|c|}
\hline Parameters & Before UV & After UV \\
\hline TE(Hartree) & -1584.96253 & -1583.70425 \\
\hline AZE(Kcal/Mol) & 215.35014 & 201.52769 \\
\hline \multirow{3}{*}{$\operatorname{RCs}(\mathbf{G H z})$} & 0.21044 & 0.21625 \\
\hline & 0.16587 & 0.18408 \\
\hline & 0.12202 & 0.12481 \\
\hline \multicolumn{3}{|l|}{ S(Cal/Mol- $\left.{ }^{\circ} \mathbf{K}\right)$} \\
\hline Total & 168.1 & 161.149 \\
\hline Transational & 43.714 & 43.698 \\
\hline Vibrational & 35.577 & 35.424 \\
\hline Rotational & 88.809 & 82.027 \\
\hline \multicolumn{3}{|l|}{$\mathrm{Cv}\left(\mathrm{Cal} / \mathrm{Mol}^{\circ}{ }^{\circ} \mathrm{K}\right)$} \\
\hline Total & 96.61 & 92.102 \\
\hline Transational & 2.981 & 2.981 \\
\hline Vibrational & 2.981 & 2.981 \\
\hline Rotational & 90.648 & 86.141 \\
\hline $\mathrm{NRP}(\mathrm{eV})$ & $7.7 * 10^{4}$ & $7.2^{*} 10^{4}$ \\
\hline TDM(Debye) & 5.72 & 8.99 \\
\hline E LUMo $\left._{\text {LV }}\right)$ & -3.06 & -4.14 \\
\hline Eномо $(\mathrm{eV})$ & -6.80 & -6.80 \\
\hline ELUMO/номо $_{\text {LeV) }}$ & 3.75 & 2.66 \\
\hline IP(eV) & 3.06 & 0.15 \\
\hline EA $(e V)$ & 6.80 & 0.25 \\
\hline$\chi(\mathrm{eV})$ & 1.87 & 1.33 \\
\hline$\kappa(\mathrm{eV})$ & -4.93 & -5.47 \\
\hline$\phi(\mathrm{eV})$ & 6.49 & 11.27 \\
\hline$S\left(\mathrm{eV}^{-1}\right)$ & 0.53 & 0.75 \\
\hline
\end{tabular}

Quantum-chemistry researchers apply FMOs analyses to expect reactivities as well as to justify reaction-pathway mechanisms [37]. Reaction pathways, as well as reactivity, are 
related to how intensive HOMOs/LUMOs interactions are. Outstanding physical features are derived such interactions like ionized-potential $I P=-E_{\text {номо }}$, electron-affinity $E A=$ $-E_{\text {LUMO }}$, chemical-potential $\chi=\left(E_{\text {LUMO }}+E_{\text {HOMO }}\right) / 2$, hardness $\kappa=\left(E_{\text {LUMO }}-E_{\text {HOMO }}\right) / 2$, electrophile-index $\phi=\chi^{2} / 2 \kappa$ and softness $S=1 / \kappa 24$. m-CP molecule has a high dipolemoment (5.72 D) and HOMO/LUMO offset of $3.75 \mathrm{eV}$ (see Fig.4(a)) which may compete $\mathrm{Si}$ $\&$ Ge based-solar cells band offsets (3.9and $2.8 \mathrm{eV}$, respectively) [25]. Such evidence stimulates researchers' insights to fabricate new m-CP based solar devices and check their efficiencies. Fig. 5-a presents the calculated DOS chart for $\mathrm{m}-\mathrm{CP}$.

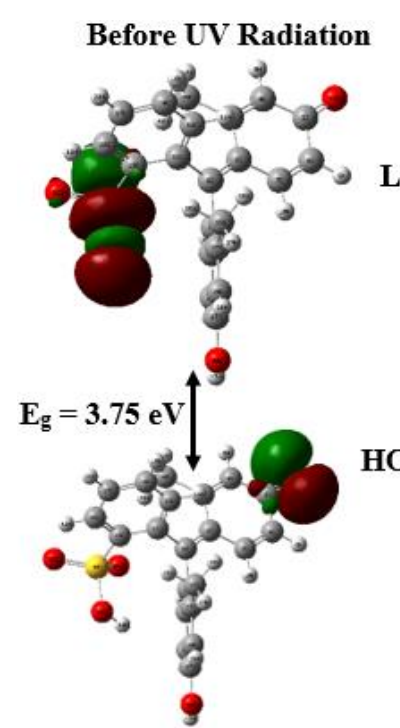

(a)
After UV Radiation

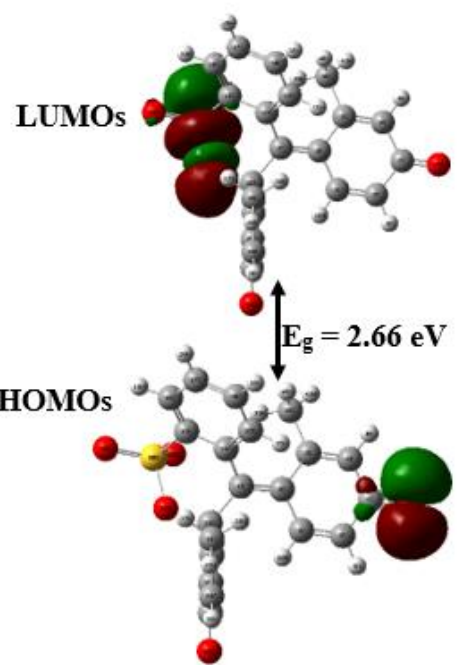

(b)

Figure 4. HOMO/LUMO offsets for m-CP (a) before, (b) after UV exposure.

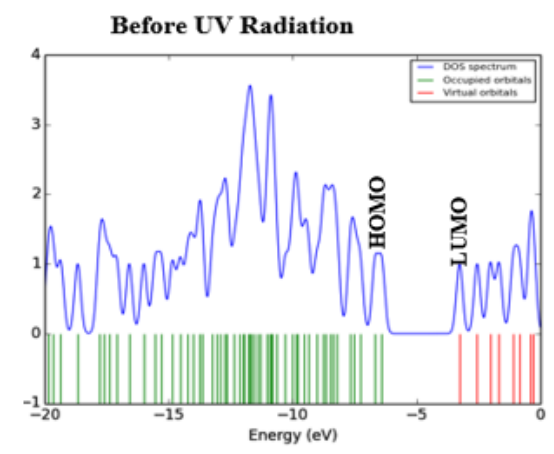

(a)

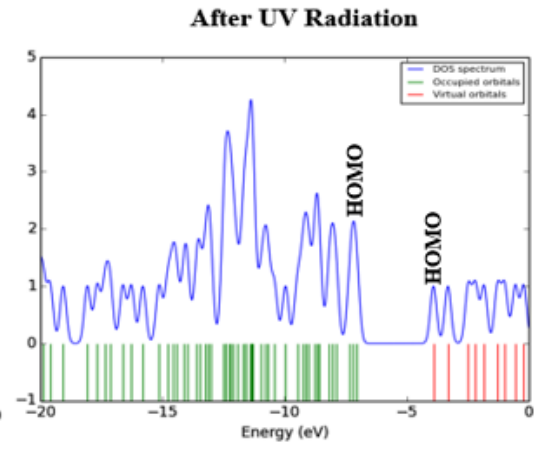

(b)

Figure 5. DOS charts form-CP (a) before, (b) after UV exposure.

\subsection{Influence $U V$ exposure on m-CP optical response.}

Authors inspect UV exposure impact on m-CP physical features. Since m-CP holds over many terminal hydrogens (see Fig. 1), which can be decidedly influenced by UV radiation, authors speculate that UV photons attacked hydrogens associated with both $\mathrm{OH}$ groups promoting hydrogen proton splitting. Such an unexpected change raises the electron density around $\mathrm{O}$ atoms [38]. First, the lone pair of $\mathrm{O}_{44}$ interact with contiguous $\mathrm{C}_{5}$ form a $\pi$ conjugation. Second, the other lone pair of $\mathrm{O}_{33}$ remains free resonating through the whole $\mathrm{SO}_{3}$ group. The UV exposure effect can be noticed from listed computed thermochemistry data (cf. Table 3). Irradiated m-CP dipole-moment has improved up to $8.99 \mathrm{D}$, and HOMO LUMO offset has lowered to $2.66 \mathrm{eV}$. Such surprising influence on bandgap offset and dipole-moment will trigger an efficient electron rush process through FMOs. Consequently, irradiated m-CP 
reserve primer position as outstanding window layer for solar cell applications [39-42]. Fig. 4b presents calculated irradiated m-CP HOMO/LUMO offset while Fig. 5-b shows computed DOS chart for irradiated $\mathrm{m}-\mathrm{CP}$ as well.

\subsection{Polarizability/hyperpolarizability calculations.}

Polarizabilities and $1^{\text {st }}$ hyperpolarizabilities are computed to inspect NLO response for $\mathrm{m}-\mathrm{CP}$ before and after UV exposure through WB97XD6-311G calculation level. Meanpolarizability $\left[\alpha_{\text {tot }}\right]$, polarizability anisotropy $[\Delta \alpha]$ and $1^{\text {st }}$ hyperpolarizability $\left[\beta_{\text {tot }}\right]$ are computed by later mathematical formalism [43]:

$$
\begin{aligned}
& \alpha_{\mathrm{tot}}=\frac{1}{3}\left(\alpha_{\mathrm{xx}}+\alpha_{\mathrm{yy}}+\alpha_{\mathrm{zz}}\right) \\
& \Delta \alpha=\sqrt{\frac{1}{2}\left(\left(\alpha_{x x}-\alpha_{y y}\right)^{2}+\left(\alpha_{y y}-\alpha_{z z}\right)^{2}+\left(\alpha_{z z}-\alpha_{x x}\right)^{2}+6 \alpha_{y z}^{2}+6 \alpha_{x y}^{2}+6 \alpha_{x z}^{2}\right)^{\frac{1}{2}}} \\
& \beta_{t o t}=\sqrt{\left(\beta_{x y y}+\beta_{x z z}+\beta_{x x x}\right)^{2}+\left(\beta_{y z z}+\beta_{y x x}+\beta_{y y y}\right)^{2}+\left(\beta_{z x x}+\beta_{z y y}+\beta_{z z z}\right)^{2}}
\end{aligned}
$$

Enhancement in material NLO response is related right away by how TDM, $\alpha_{\text {tot }}$ and $\beta_{\text {tot }}$ values cab be increased. The higher TDM, $\alpha_{\text {tot }}$ and $\beta_{\text {tot }}$ values are, the better NLO response be. Table 5 shows $1^{\text {st }}$ hyperpolarizability $\left(\beta_{\text {tot }}\right), \Delta \alpha$ and $\alpha_{\text {tot }}$ for $\mathrm{m}-\mathrm{CP}$ before and after $\mathrm{UV}$-exposure. The $\alpha_{\text {tot }}$ and $\Delta \alpha$ values for $\mathrm{m}-\mathrm{CP}$ before and after UV exposure are $40.85^{*} 10^{-}$ ${ }^{24}, 22.69 * 10^{-24}$ and $40.25 * 10^{-24}, 28.19 * 10^{-24} \mathrm{e} . \mathrm{su}$, respectively. The $\beta_{\text {tot }}$ for $\mathrm{m}-\mathrm{CP}$ before and after UV-exposure are $11.53 * 10^{-30}$ and $1.87 * 10^{-30}$ e.su, respectively. M-CP NLO response has been lowered after UV-exposure, which may be explained by excitons formation inside irradiated $\mathrm{m}-\mathrm{CP}$. The $1^{\text {st }}$ hyperpolarizability $\left(\beta_{\text {tot }}\right)$ form-CP is 31 times above urea ( $\beta_{\text {urea }}=$ $0.3728 * 10^{-30}$ e.su). Such a magnificent NLO response nominates $\mathrm{m}-\mathrm{CP}$ material as premier in NLO technologies.

\begin{tabular}{|c|c|c|c|c|c|}
\hline \multirow[t]{2}{*}{ Parameters } & \multicolumn{2}{|c|}{ Polarizability $\left(* 10^{-24}\right.$ e.su $)$} & & \multicolumn{2}{|c|}{ Hyperpolarizability $\left(* 10^{-30}\right.$ e.su) } \\
\hline & Before UV & After UV & & Before UV & After UV \\
\hline$\alpha_{x x}$ & 361.08 & 360.46 & $\beta_{x x x}$ & 7.07 & -0.26 \\
\hline$\alpha_{x y}$ & -15.83 & -0.08 & $\beta_{x x y}$ & 5.40 & -0.28 \\
\hline$\alpha_{y y}$ & 269.81 & 277.16 & $\beta_{x y y}$ & 3.07 & -0.37 \\
\hline$\alpha_{x z}$ & -11.91 & -7.96 & $\beta_{y y y}$ & 0.91 & 2.73 \\
\hline$\alpha_{y z}$ & 24.32 & 7.82 & $\beta_{x x z}$ & 0.10 & -0.21 \\
\hline$\alpha_{z z}$ & 196.10 & 177.17 & $\beta_{x y z}$ & -0.83 & 0.20 \\
\hline$\alpha_{\text {tot }}$ & 40.85 & 40.25 & $\beta_{y y z}$ & 0.09 & -0.63 \\
\hline \multirow[t]{4}{*}{$\Delta \alpha$} & 22.69 & 23.73 & $\beta_{x z z}$ & -0.28 & 0.30 \\
\hline & & & $\beta_{y z z}$ & -0.40 & -0.15 \\
\hline & & & $\boldsymbol{\beta}_{z z z}$ & 0.73 & 0.53 \\
\hline & & & $\beta_{\text {tot }}$ & 11.53 & 1.87 \\
\hline
\end{tabular}

Table 5. Mean-polarizability $\alpha_{\text {tot }}$, polarizability anisotropy $\Delta \alpha$ and $1^{\text {st }}$ hyperpolarizability $\beta_{\text {tot }}$ for m-CP before and after UV exposure.

\subsection{ADME parameters and bioactivity.}

Swiss-ADME for estimating physicochemical descriptors. Smile notation of the m-CP is $\mathrm{Oc} 1 \mathrm{ccc}(\mathrm{c}(\mathrm{c} 1) \mathrm{C}) \mathrm{C} 1(\mathrm{OS}(=\mathrm{O})(=\mathrm{O}) \mathrm{c} 2 \mathrm{c} 1 \mathrm{cccc} 2) \mathrm{c} 1 \mathrm{ccc}(\mathrm{cc} 1 \mathrm{C}) \mathrm{O}$. m-CP's remarkable biological activity emerges from the hydroxytoluene ring plays a significant role in its antimicrobial activity. Bioavailability Radar (see Fig 6-a) demonstrates product similarity of m-CP. It is also used to anticipate characterizations like ADME, pharmacokinetics, therapeutic drug features, listed in Table 6. Six physicochemical characteristics of $\mathrm{m}-\mathrm{CP}$ have been tested, namely 
lipophilicity, size polarity, solubility, edibility, and saturation, which confirms that both isomers of $\mathrm{m}-\mathrm{CP}$ are ingested through both respiratory/gastrointestinal systems and intact skin as well [44].

Table 6. Biological activity and ADME parameters of m-CP.

\begin{tabular}{|c|c|c|c|}
\hline \multicolumn{2}{|c|}{ Physicochemical Properties } & \multicolumn{2}{|l|}{ Water Solubility } \\
\hline Formula & $\mathrm{C}_{21} \mathrm{H}_{18} \mathrm{O}_{5} \mathrm{~S}$ & $\log S[\mathrm{ESOL}]$ & -4.93 \\
\hline Molecular weight & $382.43 \mathrm{~g} / \mathrm{mol}$ & Solubility & $\begin{array}{l}4.44 * 10^{-3} \mathrm{mg} \mathrm{ml}^{-1} \\
1.16 * 10^{-5} \mathrm{~mol} / 1\end{array}$ \\
\hline No. Non-H atoms & 27 & Class & Moderately soluble \\
\hline No. Ar. Non-H atoms & 18 & $\log S$ [Ali] & -5.38 \\
\hline Fractional Csp3 & 0.14 & Solubility & $\begin{array}{l}1.60 * 10^{-3} \mathrm{mg} \mathrm{ml}^{-1} ; 4.18 \\
* 10^{-6} \mathrm{~mol} / \mathrm{l}\end{array}$ \\
\hline No. rotational bonds & 2 & Class & Mid solubility \\
\hline No. H-bond acceptors & 5 & $\log S$ [SILICOS-IT] & -6.92 \\
\hline No. H-bond donors & 2 & Solubility & $\begin{array}{l}4.61 \mathrm{e}^{*} 10^{-5} \mathrm{mg} \mathrm{ml}^{-1} ; \\
1.21 * 10^{-7} \mathrm{~mol} / 1\end{array}$ \\
\hline Molar-Refractivity & 101.50 & Class & Poor solubility \\
\hline TPSA & $92.21 \AA^{2}$ & & \\
\hline \multicolumn{2}{|l|}{ Lipophilicity } & \multicolumn{2}{|l|}{ Drug-likeness } \\
\hline $\log \left[P_{\mathrm{o} / \mathrm{w}}\right][\mathrm{iLOGP}]$ & 2.23 & Lipinski & $\mathrm{Y} ; 0$ violation \\
\hline $\log \left[P_{\mathrm{o} / \mathrm{w}}\right][\mathrm{XLOGP3}]$ & 3.75 & Ghose & $\mathrm{Y}$ \\
\hline $\log \left[P_{\mathrm{o} / \mathrm{w}}\right][\mathrm{WLOGP}]$ & 4.70 & Veber & $\mathrm{Y}$ \\
\hline $\log \left[P_{\mathrm{o} / \mathrm{w}}\right][$ MLOGP $]$ & 3.18 & Egan & $\mathrm{Y}$ \\
\hline $\log \left[P_{\mathrm{o} / \mathrm{w}}\right][$ SILICOS-IT] & 3.82 & Muegge & $\mathrm{Y}$ \\
\hline Consensus $\log \left[P_{\mathrm{o} / \mathrm{w}}\right]$ & 3.54 & Bioavailability Score & 0.55 \\
\hline \multicolumn{2}{|l|}{ Pharmacokinetics } & \multicolumn{2}{|l|}{ Medicinal Chemistry } \\
\hline GI absorption & $\mathrm{H}$ & PAINS & 1 alert: phenol_sulfite_A \\
\hline BBB permeant & Nil & Brenk & 1 alert: sulfonic_acid_1 \\
\hline P-gp substrate & $\mathrm{Y}$ & Lead likeness & $\begin{array}{l}\text { No; } 2 \text { violations: } \mathrm{MW}> \\
350, \text { XLOGP3 > } 3.5\end{array}$ \\
\hline CYP1A2 & $\mathrm{Y}$ & Synthetic accessibility & 4.03 \\
\hline CYP2C19 & $\mathrm{N}$ & & \\
\hline CYP2C9 & $\mathrm{N}$ & & \\
\hline CYP2D6 & $\mathrm{N}$ & & \\
\hline CYP3A4 & $\mathrm{N}$ & & \\
\hline $\log K_{\mathrm{p}}$ (skin permeation) & $-5.97 \mathrm{~cm} \mathrm{~s}^{-1}$ & & \\
\hline
\end{tabular}

Ar (Aromatic); Ali (Aliphatic); H(High); Y (Yes); N(No)
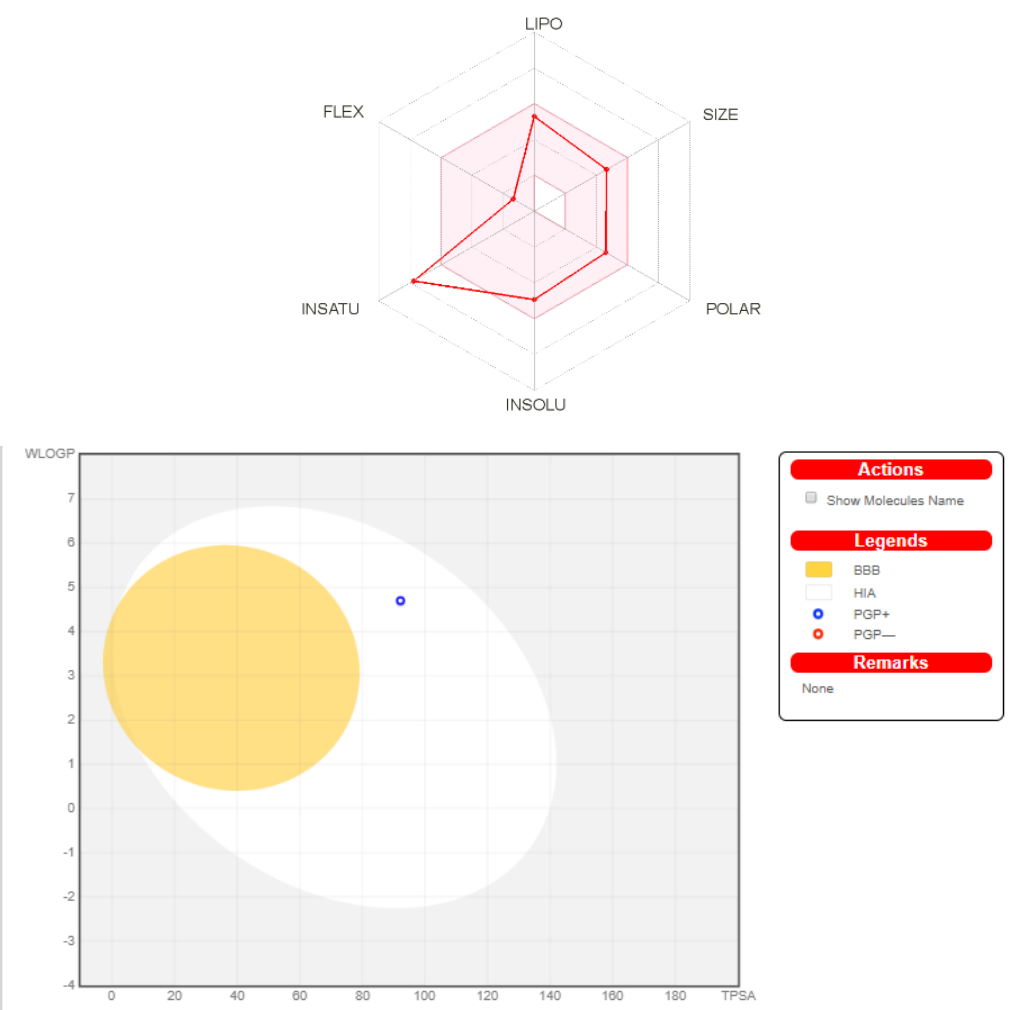

Figure 6. (a) ADME representation (b) Boiled-Egg predictive model for m-CP. 
$\mathrm{m}-\mathrm{CP}$ is reported to have a high absorption of Gastrointestinal (GI) in Pharmacokinetics. Definite results suggest that $\mathrm{m}-\mathrm{CP}$ largely transmitted all cross body after uptake. $\mathrm{m}$ $\mathrm{CP}$ usually mixes with inorganic sulfate and glucuronic acid, which excretes as urine agonists [45]. It has BBB zero, suggesting that they are strongly hydrophilic, polar chemicals to predict bowel permeation.

$\mathrm{m}$-CP physicochemical spectrum adapted as a pink zone on each axis to be a well-defined descriptor of the drug, which yields a predictive model, Boiled-Egg (see Fig. 6-b). Molecules that are favorably absorbed by the gastrointestinal tract are presented as white space, while those who penetrate the brain are presented by yellow (yolk) one.

\subsection{Pharmaco-kinetic properties and QSAR studies.}

Bioactivity provides information on the qualitative assessment of physicochemical properties that make an oral drug molecule possible. The bioavailability of the title molecule is $55 \%$ as $\mathrm{m}-\mathrm{CP}$ isomers are specifically used in bactericides or disinfectants as the active ingredient [46]. Both $\rho$-cresyl and $\rho$-cresyl sulfate, namely cresol metabolites, showed human protein/albumin binding ratio of approximately 13-20\% [47]. Table 7 represents $\mathrm{m}-\mathrm{CP}$ biological activity score. Target cases with protein binding percentages and molecular lipophilicity potential for Cresol molecule are shown in Fig. 7(a\&b), which confirm that ligand interacts with the proposed nuclear reactor by $33 \%$.

Table 7. Biological activity score for m-CP.

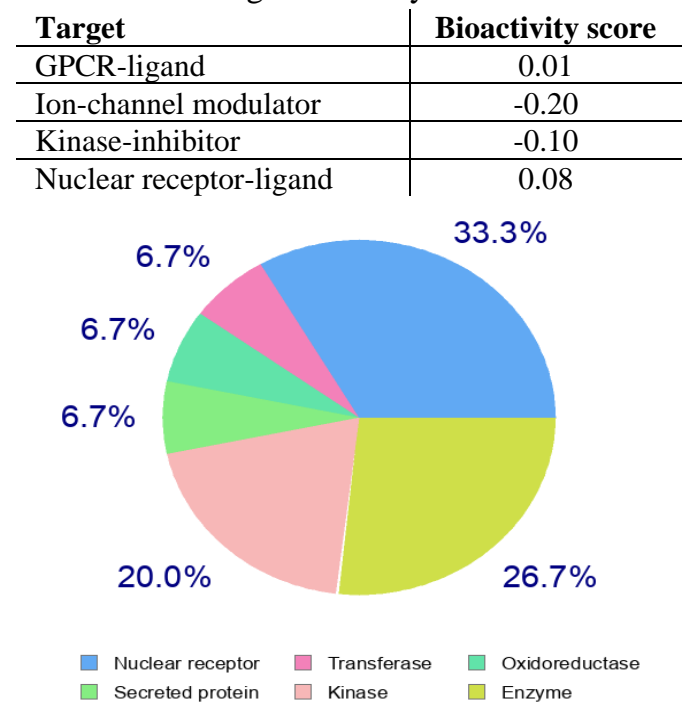

Figure 7. (a) Target cases with the percentage of protein binding for $\mathrm{m}-\mathrm{CP}$.

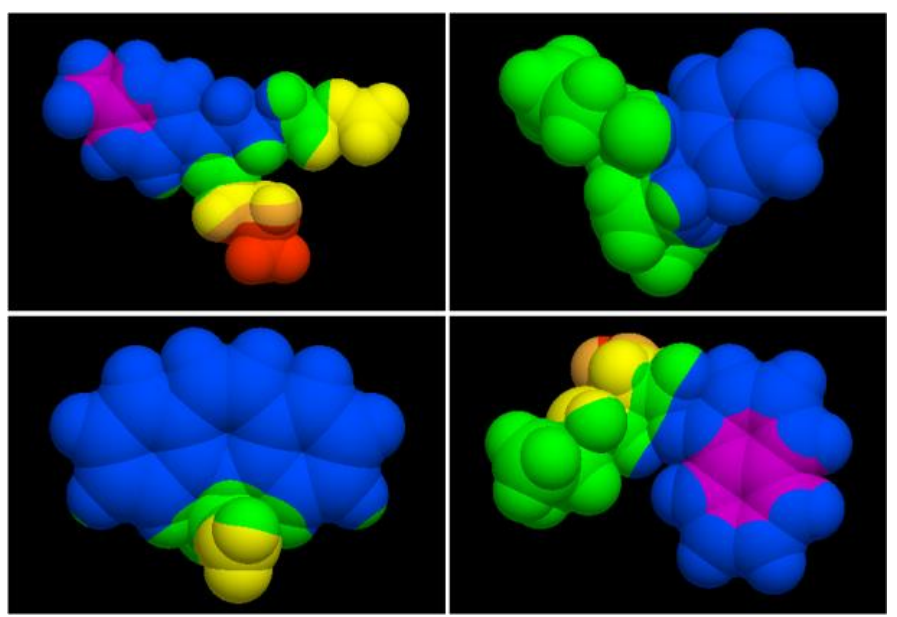

Figure 7. (b) Molecular lipophilicity potential of m-CP. 


\subsection{Micro species distribution and topological analysis.}

Especially, $\mathrm{pH}$ distribution is a useful property for drug discovery, $\log \mathrm{D}(\mathrm{pH})$ values for a series of compounds are used to calculate potential absorption sites from GI tract. Actually, $\mathrm{pH}$ is the most awaited drug discovery element. The typical logarithm of $\mathrm{pH}$ micro species presents with $\mathrm{H}$ bond donor and Cresol acceptor sites in range 0 14 is described in Fig. 8 , and $\mathrm{pH}$ calculated results are listed in Table 8 . Polar surface area (2D) of $\mathrm{m}-\mathrm{CP}$ is $83.83 \mathrm{~A}^{2}$, $\mathrm{pH}$ max is 7.40 and surface area (3D) of Van der Waals $=522.51 \mathrm{~A}^{2}$. Refractivity of $\mathrm{m}-\mathrm{CP}$ is $94.12 \mathrm{~m}^{3} \mathrm{~mol}^{-1}$ Polarizability $34.9 \AA^{3}$, Number of Rings 4, pKa (high-acidic) (high-basic) are 9.16 and -6 , respectively. Active sites that are charged negatively and positively are given both lower and higher local $\mathrm{pH}$.

Table 8. $\mathrm{pH}$ values with donor and acceptor distribution of $\mathrm{m}-\mathrm{CP}$.

\begin{tabular}{c|c|c}
$\mathbf{p H}$ value & Donor & Acceptor \\
\hline 0.00 & 2.00 & 8.00 \\
\hline 0.50 & 2.00 & 8.00 \\
\hline 1.00 & 2.00 & 8.00 \\
\hline 1.50 & 2.00 & 8.00 \\
\hline 2.00 & 2.00 & 8.00 \\
\hline 2.50 & 2.00 & 8.00 \\
\hline 3.00 & 2.00 & 8.00 \\
\hline 3.50 & 2.00 & 8.00 \\
\hline 4.00 & 2.00 & 8.00 \\
\hline 4.50 & 2.00 & 8.00 \\
\hline 5.00 & 2.00 & 8.00 \\
\hline 5.50 & 2.00 & 8.00 \\
\hline 6.00 & 2.00 & 8.00 \\
\hline 6.50 & 2.00 & 8.00 \\
\hline 7.00 & 1.99 & 8.01 \\
\hline 7.50 & 1.98 & 8.02 \\
\hline 8.00 & 1.95 & 8.05 \\
\hline 8.50 & 1.85 & 8.15 \\
\hline 9.00 & 1.59 & 8.41 \\
\hline 9.50 & 1.10 & 8.90 \\
\hline 10.00 & 0.56 & 9.44 \\
\hline 10.50 & 0.22 & 9.78 \\
\hline 11.00 & 0.07 & 9.93 \\
\hline 11.50 & 0.02 & 9.98 \\
\hline 12.00 & 0.01 & 9.99 \\
\hline 12.50 & 0.00 & 10.00 \\
\hline 13.00 & 0.00 & 10.00 \\
\hline 13.50 & 0.00 & 10.00 \\
\hline 14.00 & 0.00 & 10.00 \\
\hline & & \\
\hline 100
\end{tabular}

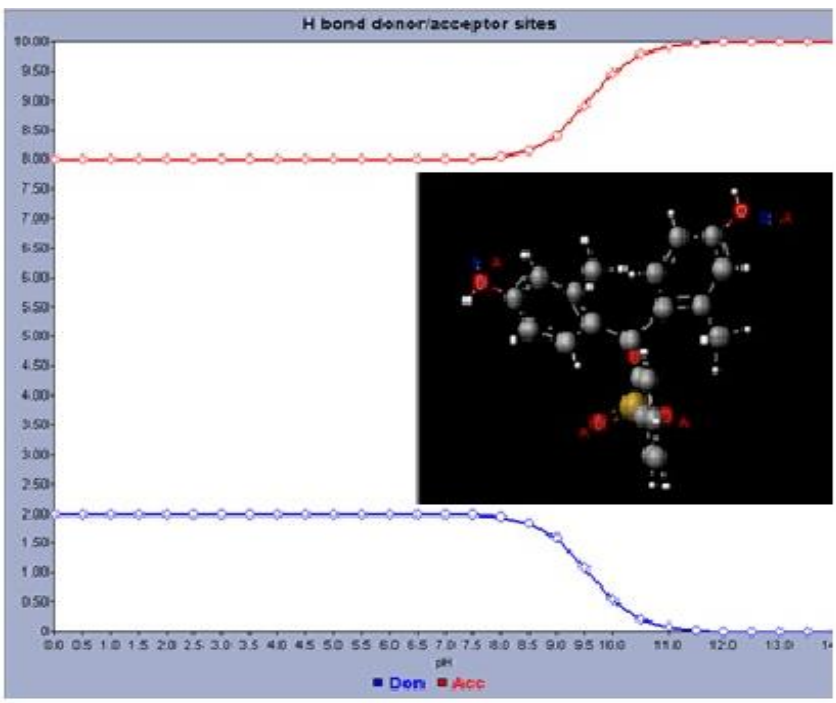

Figure 8. $\mathrm{H}$ bond donor/acceptor sites with $\mathrm{pH}$ values of $\mathrm{m}-\mathrm{CP}$. 
Table 9 represents the Topological analysis of $\mathrm{m}-\mathrm{CP}$ molecule. Phenol sulfonphthale in or $\mathrm{m}-\mathrm{CP}$ is a $\mathrm{pH}$ sensor widely used in cell biology laboratories, or otherwise called phenol red. Due to its poor estrogenic compound's activity, phenol sulfonphthalein is being investigated for clinical application [48].

Table 9. Topological analysis of m-CP.

\begin{tabular}{c|c|c|c|c|c|c} 
& \multicolumn{7}{c}{ Topological Analysis } \\
\hline Count & Bond & Atom & Ring & Carbo-ring & Hetro & Fuse-ring \\
\hline Total & 48 & 45 & & & & \\
\hline Aliphatic & 12 & 9 & 1 & 0 & 1 & 0 \\
\hline Aromatic & 18 & 18 & 3 & 3 & 0 & 0 \\
\hline Chain & 8 & 6 & & & & \\
\hline Ring & 22 & 21 & & & & \\
\hline Rotatory & & 2 & & & & \\
\hline carbo & & & 3 & & &
\end{tabular}

\section{Conclusions}

The theoretical research was performed $\mathrm{m}-\mathrm{CP}$, which exhibits outstanding electronic and photovoltaic properties and efficient drug-like behavior. A proper harmony between measured and predicted FTIR charts is observed. Single and double vibrational studies with predicted crystallographic data on $\mathrm{m}-\mathrm{CP}$ dye have been reported. Unusual electrostatic interactions between $\mathrm{O}_{33}$ and both $\mathrm{C}_{22}$ and $\mathrm{C}_{23}$ are observed to influence m-'CP's NLO activity. FMO transitions and DOS Map promotes the manufacture of modern $\mathrm{m}-\mathrm{CP}$ based solar devices and efficiencies. Both polarization and $1^{\text {st }}$ hyperpolarization capabilities are measured to inspect NLO response for m-CP before and after exposure to UV using WB97XD6-311G level. In addition, m-CP obey Lipinski" law of five (RO5), which means that theoretically, such compounds should not have difficulties with oral dosage. ADME, pharmacokinetic properties, $\log \mathrm{P}, \mathrm{pH}$ value, biological activity, Boiled-Egg predictive model, QSAR, lipophilicity, microorganism distribution, target binding rate, and topological measurements are studied to aid with drug discovery. According to the authors` report bio-report, $\mathrm{m}-\mathrm{CP}$ can be efficiently used as bactericides, pesticides, and disinfectants.

\section{Funding}

This research received no external funding.

\section{Acknowledgments}

This research has no acknowledgment.

\section{Conflicts of Interest}

The authors declare no conflict of interest.

\section{References}

1. Irimia-Vladu, M.; Głowacki, E.D.; Troshin, P.A.; Schwabegger, G.; Leonat, L.; Susarova, D.K.; Krystal, O.; Ullah, M.; Kanbur, Y.; Bodea, M.A.; Razumov, V.F.; Sitter, H.; Bauer, S.; Sariciftci, N.S. Indigo - A Natural Pigment for High Performance Ambipolar Organic Field Effect Transistors and Circuits. Advanced Materials 2012, 24, 375-380, https://doi.org/10.1002/adma.201102619. 
2. Abdelwahab, A.B.; El-Sawy, E.R.; Kirsch, G. Iron powder and tin/tin chloride as new reducing agents of Meerwein arylation reaction with unexpected recycling to anilines. Synthetic Communications 2020, 50, 526-538, https://doi.org/10.1080/00397911.2019.1704786.

3. Jakkampudi, S.; Konda, S.; Arman, H.; Zhao, J.C.G. Diastereodivergent Synthesis of Bridged Tetrahydroisoquinoline Derivatives Catalyzed by Modularly Designed Organocatalysts. Advanced Synthesis \& Catalysis 2020, 362, 2419-2426, https://doi.org/10.1002/adsc.202000227.

4. Elmorsy, M.R.; Su, R.; Abdel-Latif, E.; Badawy, S.A.; El-Shafei, A.; Fadda, A.A. New cyanoacetanilides based dyes as effective co-sensitizers for DSSCs sensitized with ruthenium (II) complex (HD-2). Journal of Materials Science: Materials in Electronics 2020, 31, 7981-7990, https://doi.org/10.1007/s10854-02003337-3.

5. Asif, M.; Hassanain, M.A.; Nahiduzzaman, K.M.; Sawalha, H.J.E. Smart and Sustainable Built Environment. Smart and Sustainable Built Environment 2019, 8, 34-52, https://doi.org/10.1108/SASBE-062018-0032.

6. Dinparast, L.; Hemmati, S.; Alizadeh, A.A.; Zengin, G.; Kafil, H.S.; Bahadori, M.B.; Dastmalchi, S. An efficient, catalyst-free, one-pot synthesis of $4 \mathrm{H}$-chromene derivatives and investigating their biological activities and mode of interactions using molecular docking studies. Journal of Molecular Structure 2020, 1203, https://doi.org/10.1016/j.molstruc.2019.127426.

7. Pešić, M.; Bugarinović, J.; Minić, A.; Novaković, S.B.; Bogdanović, G.A.; Todosijević, A.; Stevanović, D.; Damljanović, I. Electrochemical characterization and estimation of DNA-binding capacity of a series of novel ferrocene derivatives. Bioelectrochemistry $\quad \mathbf{2 0 2 0}$ 132, https://doi.org/10.1016/j.bioelechem.2019.107412.

8. Saji, R.S.; Prasana, J.C.; Muthu, S.; George, J.; Kuruvilla, T.K.; Raajaraman, B.R. Spectroscopic and quantum computational study on naproxen sodium. Spectrochimica Acta Part A: Molecular and Biomolecular Spectroscopy 2020, 226, https://doi.org/10.1016/j.saa.2019.117614.

9. El-Mansy, M. FT-IR, FT-Raman Spectra and Ab Initio HF, DFT Vibrational Analysis of P-methyl acetanilide. Journal of Applied Sciences Research 2009, 5, 1977-1987.

10. El-Mansy, M.A.M.; El-Nahass, M.M.; Khusayfan, N.M.; El-Menyawy, E.M. DFT approach for FT-IR spectra and HOMO-LUMO energy gap for $\mathrm{N}$-(p-dimethylaminobenzylidene)-p-nitroaniline (DBN). Spectrochimica Acta Part A: Molecular and Biomolecular Spectroscopy 2013, 111, 217-222, https://doi.org/10.1016/j.saa.2013.04.018.

11. El-Mansy, M.A.M.; Ismail, M.M. On the spectroscopic analyses of 3-(4-Hydroxy-1-methyl-2-oxo-1,2dihydro-quinolin-3-yl)-2-nitro-3-oxo-propionic acid (HMQNP). Spectrochimica Acta Part A: Molecular and Biomolecular Spectroscopy 2015, 135, 704-709, https://doi.org/10.1016/j.saa.2014.07.033.

12. El-Mansy, M.A.M.; Yahia, I.S. Spectroscopic notes of Methyl Red (MR) dye. Spectrochimica Acta Part A: Molecular and Biomolecular Spectroscopy 2014, 130, 59-63, https://doi.org/10.1016/j.saa.2014.03.113.

13. El-Nahass, M.M.; Kamel, M.A.; El-Barbary, A.A.; El-Mansy, M.A.M.; Ibrahim, M. FT-IR spectroscopic analyses of 3-Methyl-5-Pyrazolone (MP). Spectrochimica Acta Part A: Molecular and Biomolecular Spectroscopy 2013, 111, 37-41, https://doi.org/10.1016/j.saa.2013.03.072.

14. Ibrahim, M.; El-Barbary, A.A.; El-Nahass, M.M.; Kamel, M.A.; El-Mansy, M.A.M.; Asiri, A.M. On the spectroscopic analyses of (E)-3-(dicyclopropyl methylene)-dihydro-4-[1-(2,5 dimethylfuran-3-yl) ethylidene]furan-2,5-dione. Spectrochimica Acta Part A: Molecular and Biomolecular Spectroscopy 2012, 87, 202-208, https://doi.org/10.1016/j.saa.2011.11.039.

15. Ibrahim, M.; El-Nahass, M.M.; Kamel, M.A.; El-Barbary, A.A.; Wagner, B.D.; El-Mansy, M.A.M. On the spectroscopic analyses of thioindigo dye. Spectrochimica Acta Part A: Molecular and Biomolecular Spectroscopy 2013, 113, 332-336, https://doi.org/10.1016/j.saa.2013.05.014.

16. Ismail, M.M.; Morsy, G.M.; Mohamed, H.M.; El-Mansy, M.A.M.; Abd-Alrazk, M.M.A. FT-IR spectroscopic analyses of 4-hydroxy-1-methyl-3-[2-nitro-2-oxoacetyl-2(1H)quinolinone (HMNOQ). Spectrochimica Acta Part A: Molecular and Biomolecular Spectroscopy 2013, 113, 191-195, https://doi.org/10.1016/j.saa.2013.04.117.

17. Soliman, H.S.; Eid, K.M.; Ali, H.A.M.; Atef, S.M.; El-Mansy, M.A.M. Vibrational spectroscopic analysis of 2-chloro-5-(2,5-dimethoxy-benzylidene)-1,3-diethyl-dihydro-pyrimidine-4,6 $(1 \mathrm{H}, 5 \mathrm{H})$-dione. Spectrochimica Acta Part A: Molecular and Biomolecular Spectroscopy 2012, 97, 1079-1084, https://doi.org/10.1016/j.saa.2012.07.104.

18. Soliman, H.S.; Eid, K.M.; Ali, H.A.M.; El-Mansy, M.A.M.; Atef, S.M. FT-IR spectroscopic analyses of 2(2-furanylmethylene) propanedinitrile. Spectrochimica Acta Part A: Molecular and Biomolecular Spectroscopy 2013, 105, 545-549, https://doi.org/10.1016/j.saa.2012.12.051.

19. Hassnin, H. Nucleophilic substitution and ring transformation reactions with 4-chloro-6-ethyl-3nitropyrano[3,2-c]quinoline-2,5(6H)-dione. Arkivoc 2012, $2012, \quad$ 384-397, http://dx.doi.org/10.3998/ark.5550190.0013.634.

20. Frisch, M.J.; Schlegel, G.W.T.H.B.; Scuseria, G.E.; Robb, M.A.; Cheeseman, J.R.; Montgomery, Jr.J.A.; Vreven, T.; Kudin, K.N.; Burant, J.C.; Millam, J.M.; Iyengar, S.S.; Tomasi, J.; Barone, V.; Mennucci, B.; Cossi, M.; Scalmani, G.; Rega, N.; Petersson, G.A.; Nakatsuji, H.; Hada, M.; Ehara, M.; Toyota, K.; Fukuda, R.; Hasegawa, J.; Ishida, M.; Nakajima, T.; Honda, Y.; Kitao, O.; Nakai, H.; Klene, M.; Li, X.; Knox, J.E.; 
Hratchian, H.P.; Cross, J.B.; Adamo, C.; Jaramillo, J.; Gomperts, R.; Stratmann, R.E.; Yazyev, O.; Austin, A.J.; Cammi, R.; Pomelli, C.; Ochterski, J.W.; Ayala, P.Y.; Morokuma, K.; Voth, G.A.; Salvador, P.; Dannenberg, J.J.; Zakrzewski, V.G.; Dapprich, S.; Daniels, A.D.; Strain, M.C.; Farkas, O.; Malick, D.K.; Rabuck, A.D.; Raghavachari, K.; Foresman, J.B.; Ortiz, J.V.; Cui, Q.; Baboul, A.G.; Clifford, S.; Cioslowski, J.; Stefanov, B.B.; Liu, G.; Liashenko, A.; Piskorz, P.; Komaromi, I.; Martin, R.L.; Fox, D.J.; Keith, T.; AlLaham, A.M.; Peng, C.Y.; Nanayakkara, A.; Challacombe, M.; Gill, P.M.W.; Johnson, B.; Chen, W.; Wong, M.W.; Gonzalez, C.; Pople, J.A. Inc., Wallingford CT 2009.

21. Frisch, A.; Dennington, R.; Keith, T.; Millam, J.; Nielsen, A.; Holder, A.; Hiscocks, J. Gaussian Inc. Wallingford, CT, USA 2009

22. El-Mansy, M.A.M. Quantum chemical studies on structural, vibrational, nonlinear optical properties and chemical reactivity of indigo carmine dye. Spectrochimica Acta Part A: Molecular and Biomolecular Spectroscopy 2017, 183, 284-290, https://doi.org/10.1016/j.saa.2017.04.047.

23. Xiao, F.; Yao, S.; Wang, J.; Wei, J.; Amirkhanian, S. Physical and chemical properties of plasma treated crumb rubbers and high temperature characteristics of their rubberised asphalt binders. Road Materials and Pavement Design 2020, 21, 587-606, https://doi.org/10.1080/14680629.2018.1507922.

24. Chaudhary, J.P.; Kholiya, F.; Vadodariya, N.; Budheliya, V.M.; Gogda, A.; Meena, R. Carboxymethylagarose-based multifunctional hydrogel with super stretchable, self-healable having film and fiber forming properties. Arabian Journal of Chemistry 2020, 13, 1661-1668, https://doi.org/10.1016/j.arabjc.2017.12.034.

25. Sureshkumar, B.; Mary, Y.S.; Panicker, C.Y.; Suma, S.; Armaković, S.; Armaković, S.J.; Van Alsenoy, C.; Narayana, B.J.A.J.o.C. Quinoline derivatives as possible lead compounds for anti-malarial drugs: spectroscopic, DFT and MD study. Arabian Journal of chemistry 2020, 13, 632-648. https://doi.org/10.1016/j.arabjc.2017.07.006

26. Gulácsi, M.; El-Mansy, M.A.M.; Gulácsi, Z. Electron-phonon interactions in conducting polymers. Philosophical Magazine Letters 2016, 96, 67-75, https://doi.org/10.1080/09500839.2016.1150611.

27. Baú, J.P.T.; Anizelli, P.R.; de Santana, H.; da Costa, M.F.; Zaia, D.A.M. An experimental and theoretical vibrational study of the interaction of cytosine and uracil with artificial seawaters: A prebiotic chemistry experiment. Vibrational Spectroscopy 2019, 101, 92-99, https://doi.org/10.1016/j.vibspec.2019.02.002.

28. Salih, T.; Salih, H.A.J.A.-M.J.f.P.S. In Silico Design and Molecular Docking Studies of Carbapenem Analogues Targeting Acinetobacter baumannii PBP1A Receptor. Iraq Academic Scientific Journals 2020, 20 .

29. Koepsell, H. Organic Cation Transporters in Health and Disease. Pharmacol Rev 2020, 72, 1-45. https://doi.org/10.1124/pr.118.015578.

30. Arrousse, N.; Salim, R.; Kaddouri, Y.; zarrouk, A.; Zahri, D.; Hajjaji, F.E.; Touzani, R.; Taleb, M.; Jodeh, $\mathrm{S}$. The inhibition behavior of two pyrimidine-pyrazole derivatives against corrosion in hydrochloric solution: Experimental, surface analysis and in silico approach studies. Arabian Journal of Chemistry 2020, 13, 59495965, https://doi.org/10.1016/j.arabjc.2020.04.030.

31. Motamarri, P.; Das, S.; Rudraraju, S.; Ghosh, K.; Davydov, D.; Gavini, V. DFT-FE - A massively parallel adaptive finite-element code for large-scale density functional theory calculations. Computer Physics Communications 2020, 246, https://doi.org/10.1016/j.cpc.2019.07.016.

32. Lgaz, H.; Salghi, R.; Masroor, S.; Kim, S.-H.; Kwon, C.; Kim, S.Y.; Yang, Y.-J.; Chung, I.-M. Assessing corrosion inhibition characteristics of hydrazone derivatives on mild steel in $\mathrm{HCl}$ : Insights from electronicscale DFT and atomic-scale molecular dynamics. Journal of Molecular Liquids 2020, 308, https://doi.org/10.1016/j.molliq.2020.112998.

33. Rajamani, P.; Vijayakumar, V.; Nagaraaj, P.; Sundaraganesan, N. Synthesis, Characterization, Spectroscopic, DFT and Molecular Docking Studies of 3-(3,4-Dihydroxyphenyl)-1-Phenyl-3(Phenylamino)Propan-1-One. Polycyclic Aromatic Compounds 2020, 1-21, https://doi.org/10.1080/10406638.2020.1837190.

34. Montoro, O.R.; Tortajada, J.; Lobato, Á.; Baonza, V.G.; Taravillo, M. Theoretical (DFT) and experimental (Raman and FTIR) spectroscopic study on communic acids, main components of fossil resins. Spectrochimica Acta Part A: Molecular and Biomolecular Spectroscopy 2020, 224, https://doi.org/10.1016/j.saa.2019.117405.

35. Zhang, C.; Cao, H.; Wang, C.; He, M.; Zhan, W.; Guo, Y. Catalytic mechanism and pathways of 1, 2dichloropropane oxidation over LaMnO3 perovskite: An experimental and DFT study. Journal of Hazardous Materials 2021, 402, https://doi.org/10.1016/j.jhazmat.2020.123473.

36. Yousefi, S.; Ansari, R.; Aghdasi, P.; Mozvashi, S.M. Structural and mechanical properties characterization of arsenene nanosheets under doping effect of transition metals: A DFT study. Physica E: Low-dimensional Systems and Nanostructures 2020, 124, https://doi.org/10.1016/j.physe.2020.114349.

37. Liu, A.; Liang, X.; Ren, X.; Guan, W.; Gao, M.; Yang, Y.; Yang, Q.; Gao, L.; Li, Y.; Ma, T. Recent Progress in MXene-Based Materials: Potential High-Performance Electrocatalysts. Advanced Functional Materials 2020, 30, https://doi.org/10.1002/adfm.202003437. 
38. Hamioud, F.; Tariq, S.; Batool, A.; Mubarak, A.A. Theoretical investigation on orthorhombic XMnO3 ( $\mathrm{X}=\mathrm{Nd}$, Dy and Ho) perovskite manganates using DFT. Chemical Physics Letters 2020, 760, https://doi.org/10.1016/j.cplett.2020.138005.

39. Dutta, R.; Ahmed, S.; Kalita, D.J. Theoretical design of new triphenylamine based dyes for the fabrication of DSSCs: A DFT/TD-DFT study. Materials Today Communications 2020, 22, https://doi.org/10.1016/j.mtcomm.2019.100731.

40. El-Mansy, M.; Ismail, M. Structural, conformational, optical, and nonlinear optical behavior of ethyl (6ethyl 5, 6-dihydro 4, 5-dioxo $4 \mathrm{H}$ pyrano [3, 2-c] quinolin-3yl) 2-oxoacetate (EPQOA): comparative theoretical and experimental studies. Optical Quantum Electronics 2021, 53, https://doi.org/10.1007/s11082-021-02749-7.

41. El-Mansy, M.; Osman, W.; Abdelsalam, H. The electronic and optical absorption properties of pristine, homo and hetero Bi-nanoclusters. Chemical Physics 2021, 111113, https://doi.org/10.1016/j.chemphys.2021.111113.

42. Bayoumy, A.M.; Osman, Y.O.; Elhaes, H.; Ibrahim, M.A.; El-Mansy, M.A. Effect of substitutions on the electronic properties of acetylsalicylic acid. Optical Quantum Electronics 2021, 53, https://doi.org/10.1007/s11082-020-02725-7.

43. Yaqoob, M.; Gul, S.; Zubair, N.F.; Iqbal, J.; Iqbal, M.A. Theoretical calculation of selenium N-heterocyclic carbene compounds through DFT studies: Synthesis, characterization and biological potential. Journal of Molecular Structure 2020, 1204, https://doi.org/10.1016/j.molstruc.2019.127462.

44. Çakmak, E.; Özbakır Işın, D. A theoretical evaluation on free radical scavenging activity of 3styrylchromone derivatives: the DFT study. Journal of Molecular Modeling 2020, 26, 1-11, https://doi.org/10.1007/s00894-020-04368-7.

45. Attar, T.; Messaoudi, B.; Benhadria, N.J.C.C.T. DFT Theoretical Study of Some Thiosemicarbazide Derivatives with Copper. Chemistry and Chemical Technology 2020, 14, 20-25, https://doi.org/10.23939/chcht14.01.020.

46. Drauch, V.; Ibesich, C.; Vogl, C.; Hess, M.; Hess, C. In-vitro testing of bacteriostatic and bactericidal efficacy of commercial disinfectants against Salmonella Infantis reveals substantial differences between products and bacterial strains. International Journal of Food Microbiology 2020, 328, https://doi.org/10.1016/j.ijfoodmicro.2020.108660.

47. Naumovski, V.; Kimble, B.; Laurenti, D.; Nammi, S.; Norimoto, H.; Chan, K. Polysaccharide peptide (PSP) extract from Coriolus versicolor decreased Tmax of tamoxifen and maintained biochemical serum parameters, with no change in the metabolism of tamoxifen in the rat. Research Square 2020, https://doi.org/10.3390/medicines7060035.

48. Luo, X.; Lim, L.-T. An inkjet-printed sulfonephthalein dye indicator array for volatile amine detection. Journal of Food Science 2020, 85, 442-454, https://doi.org/10.1111/1750-3841.15020. 